\title{
The stellar content of the XMM-Newton slew survey ${ }^{\star}$
}

\author{
S. Freund, J. Robrade, P. C. Schneider, and J. H. M. M. Schmitt
}

Hamburger Sternwarte, Universität Hamburg, 21029 Hamburg, Germany
e-mail: sebastian.freund@uni-hamburg.de, jrobrade@hs.uni-hamburg.de, cschneider@hs.uni-hamburg.de

Received 27 September 2017 / Accepted 14 December 2017

\begin{abstract}
Aims. We present a detailed analysis of the stellar content of the current version of the XMM-Newton slew survey (XMMSL2). Methods. Since stars emit only a small fraction of their total luminosity in the X-ray band, the stellar XMMSL2 sources ought to have relatively bright optical counterparts. Therefore the stellar identifications were obtained by an automatic crossmatch of the XMMSL2 catalog with the first Gaia data release (Gaia DR1), 2MASS, and Tycho2 catalogs. The reliability of this procedure was verified by a comparison with the individually classified Einstein Observatory medium sensitivity survey X-ray sources and by a crossmatch with the Chandra Source Catalog.

Results. We identify 6815 of the 23252 unique XMMSL2 sources to be stellar sources, while 893 sources are flagged as unreliable. For every counterpart a matching probability is estimated based upon the distance between the XMMSL2 source and the counterpart. Given this matching probability the sample is expected to be reliable to $96.7 \%$ and complete to $96.3 \%$. The sample contains stars of all spectral types and luminosity classes, and late-type dwarfs have the largest share. For many stellar sources the fractional contribution of the X-ray band to the total energy output is found above the saturation limit of previous studies $\left(L_{\mathrm{X}} / L_{\mathrm{bol}}=10^{-3}\right)$, because the XMMSL2 sources are more affected by flares owing to their short exposure times of typically $6 \mathrm{~s}$. A comparison with the second ROSAT all-sky survey (2RXS) source catalog shows that about $25 \%$ of the stellar XMMSL2 sources are previously unknown X-ray sources. The results of our identification procedure can be accessed via VizieR.
\end{abstract}

Key words. stars: activity - X-ray: stars - methods: miscellaneous

\section{Introduction}

Catura et al. (1975) were the first to detect coronal X-ray emission from a star other than the Sun in the bright active binary system Capella, albeit at a level much brighter than typical solar X-ray emission levels. Later, X-ray observations with the Einstein Observatory (Vaiana et al. 1981) and then with ROSAT showed X-ray emission to be ubiquitous for almost all types of stars (Schmitt et al. 1995; Schmitt 1997; Huensch et al. 1998a,b).

The X-ray properties of stars are usually investigated either by pointed observations of selected X-ray sources - for example, currently with the XMM-Newton (Jansen et al. 2001) and Chandra space telescopes or via all-sky surveys such as the ROSAT all-sky survey (RASS; Voges et al. 1999) or, in the future, the eROSITA all-sky survey (Merloni et al. 2012; Predehl 2017). Such all-sky surveys have the advantage of delivering large samples of X-ray sources that are not biased by the selection of specific sources or specific sky regions. The same applies to the XMM-Newton slew survey (XMMSL; Saxton et al. 2008), which delivers data in a spectral range identical to the upcoming eROSITA survey. The XMMSL, however, is somewhat special in the context of X-ray surveys. The XMM-Newton satellite also collects X-ray data while slewing from one pointed observation to the next and these data form the basis of the XMMSL, which is regularly updated with the mission.

Naturally, in contrast to true all-sky survey such a survey is very inhomogeneous, but in its current version (XMMSL2) the

\footnotetext{
* Catalog of the stellar XMMSL2 sources is only available at the CDS via anonymous ftp to cdsarc.u-strasbg.fr (130.79.128.5) or via http://cdsarc.u-strasbg.fr/viz-bin/qcat?]/A+A/614/A125
}

XMMSL already covers $84 \%$ of the sky and includes 29393 detections of 23252 unique X-ray sources. We are specifically interested in the stellar content of the XMMSL, hence our task at hand is the development of a procedure that distinguishes stellar sources in the XMMSL from other classes of X-ray emitters such as galaxy clusters and active galactic nuclei (AGN) as reliably and completely as possible. Because of the large number of XMMSL2 sources, this identification process can obviously not be carried out individually by hand, rather an automatic method is required that uses the known properties of stellar (coronal) Xray sources.

Stellar X-ray sources are relatively faint, when measured in terms of the fractional contribution of the X-ray band to the total energy output, i.e., the $L_{\mathrm{X}} / L_{\mathrm{bol}}$-ratio. For example, early-type stars typically satisfy $L_{\mathrm{X}} / L_{\mathrm{bol}} \approx 10^{-7}$ (Pallavicini et al. 1981; Berghoefer et al. 1997) and their X-ray emission is generated through radiative instabilities in their radiatively driven stellar winds. In contrast, the X-ray emission observed from late-type, cool stars is produced in hot coronae, and magnetic fields are thought to play a fundamental role for the coronal physics of stars (Pevtsov et al. 2003). The observed X-ray luminosities of late-type stars vary enormously, both in individual cases and in a sample of stars. In the case of flares the X-ray flux can increase by orders of magnitude over timescales of minutes to hours. Also, similar to the solar cycle late-type stars may show modulated X-ray emission on timescales of years related to activity cycles (Hempelmann et al. 2003; Favata et al. 2008; Ayres 2009; Robrade et al. 2012), in addition the X-ray flux of a given star may vary on the timescale of rotation on typically a timescale of a few days and possibly longer. As a class, late-type dwarfs show a rather well-defined maximum fractional X-ray emission 
of about $L_{\mathrm{X}} / L_{\mathrm{bol}}=10^{-3}$ during so-called quasi-quiescent periods, i.e., during times without obvious strong flares (Vilhu 1984; Agrawal et al. 1986; Fleming et al. 1988; Pallavicini et al. 1990). A similarly well-defined lower limit does not exist, but Schmitt (1997) showed the existence of a minimum X-ray surface flux of about $10^{4} \mathrm{erg} \mathrm{s}^{-1} \mathrm{~cm}^{-2}$ for dwarf stars, which results in a $L_{\mathrm{X}} / L_{\mathrm{bol}} \approx 10^{-7}-10^{-6}$ for solar analogs.

Stars off the main sequence also show X-ray emission and the X-ray luminosity can be very high, especially for giants that are part of a binary system, for example, RS CVn systems and related systems (Walter et al. 1978; Dempsey et al. 1993). However, little to no X-ray emission is found for red giants beyond the so-called dividing line (Linsky \& Haisch 1979; Haisch et al. 1991; Huensch et al. 1996).

The low fractional X-ray luminosity stellar X-ray sources implies that counterparts of these sources ought to be relatively bright in the optical band. Hence, any star, detected for example in the XMMSL, will also be detected in an optical survey of sufficient sensitivity. In this context the currently operating Gaia mission (Gaia Collaboration 2016b) is particularly relevant, since Gaia will eventually produce a complete optical catalog down to a magnitude of 20 as well as parallaxes, which allows easy distinction of nearby stellar sources from more distant Galactic and extragalactic sources. In November 2016 the first data release of the Gaia optical all-sky survey was issued by the Gaia Collaboration (2016a, Gaia DR1), and we can therefore start to tap the Gaia potential in our effort to identify the stellar XMMSL sources by a crossmatch with the Gaia DR1.

The plan of our paper is then as follows. In Sect. 2 we present the properties of the XMMSL and the Gaia DR1 catalog along with the complimentary catalogs used in this paper. In Sect. 3 we describe our matching procedure and estimate the expected completeness and reliability of our stellar identification based upon the matching probability of the individual counterparts. We present our results and compare our stellar identifications with those of Saxton et al. (2008) in Sect. 4. Additionally, we test the reliability of our automatic matching procedure by applying it to the Extended Medium-Sensitive Survey (EMSS; Gioia et al. 1990; Stocke et al. 1991) of the Einstein Observatory, whose sources have been individually classified by spectroscopy, and by performing a crossmatch with the Chandra Source Catalog. In Sect. 5 we compare the X-ray fluxes of the stellar XMMNewton slew survey sources with the corresponding fluxes of the second ROSAT all-sky survey (2RXS) source catalog (hereafter RASS catalog, Boller et al. 2016). The properties of the stellar sample of the XMMSL2 sources are presented in Sect. 6 and we draw our conclusions in Sect. 7.

\section{Catalog suite}

We first provide short descriptions of the various catalogs used in this paper.

\subsection{XMMSL catalog}

For our stellar identifications, we used the clean version of the XMMSL2 catalog as the X-ray input catalog, which we refer to as XMMSL2 catalog hereafter. For a detailed description of the catalog and its creation, we refer to Saxton et al. (2008), who describe all methods of the production of the first XMM-Newton slew survey catalog in detail; these methods are very similar to those of the XMMSL2 catalog. Briefly, this catalog contains detections with a detection likelihood of $D E T \_M L>10.5$ in general and of $D E T \_M L>15.5$ for sources with higher than the usual background. The positional accuracy is typically about 8 arcsec and, according to Saxton et al. (2008), about $4 \%$ of the sources detected in the full band (as well as $0.7 \%$ and $9 \%$ of the sources detected in the soft and hard band, respectively) are spurious.

All XMM-Newton slews are treated individually during the creation of the XMMSL2 catalog, such that every X-ray detection leads to a new entry in the catalog, even if the same source has been detected in a previous slew. In a second step, detections lying within 30 arcsec in different slews are then considered to be multiple detections of the same source and are given the same source name. Therefore, the 29393 XMMSL2 detections actually come from 23252 unique X-ray sources. For our identification of stars, we only used the unique XMMSL2 sources and coordinates of the detection with the highest detection likelihood. Further, we used the median X-ray flux for sources with multiple detections.

In the XMMSL2 catalog the source count rates are given in three different energy bands, i.e., the total band $(0.2-12 \mathrm{keV})$, soft band (0.2-2 keV), and hard band (2-12 keV).

\subsection{Gaia $D R 1$}

The Gaia DR1 catalog contains the positions and $G$ band magnitudes of 1.1 billion sources. For a subset of 2 million stars, parallaxes and proper motions were calculated from information provided by the HIPPARCos and Tycho 2 catalogs; this subset is called the Tycho-Gaia astrometric solution (TGAS). The positional and photometric uncertainties of all catalog entries are negligible compared to the uncertainties in the X-ray data (better than 10 mas and $0.03 \mathrm{mag}$, respectively).

Unfortunately, the Gaia DR1 catalog has a only preliminary character (Gaia Collaboration 2016a), and for our purpose, the relevant known limitations are its incompleteness for very bright sources $\$ 7 \mathrm{mag}$, sources with high proper motion, extremely blue or red sources, and sources located in dense areas on the sky and or in binary systems. To overcome these limitations and to obtain color information for spectral type classification, we considered complementary catalogs.

\subsection{Complementary catalogs}

To obtain colors and hence spectral types for the X-ray counterparts, we used the 2MASS catalog (Skrutskie et al. 2006), i.e., an infrared catalog that is particularly useful for stars of late spectral type (cf. Fig. 1), and the Tycho2 catalog (Høg et al. 2000) to identify the brighter sources. In addition, we considered information provided by the Bright Star catalog (Hoffleit \& Jaschek 1991) and the catalog by Lépine \& Gaidos (2011) of bright M dwarfs (Lepine catalog). In the following we describe the matching procedure only for the Gaia DR1, 2MASS, and Tycho2 catalogs, which provide the vast majority of our stellar identifications, but the presented method was also applied to the BrightStar and the Lepine catalog and appropriate matching distances and probabilities were estimated for these catalogs.

We expect essentially all stellar XMMSL2 sources to have a 2MASS counterpart. Since the completeness of the 2MASS catalog is $>99 \%$, our procedure is not influenced by the incompleteness of the catalogs used for the identification.

\section{Data analysis}

Our matching procedure is based upon the angular distance between the XMMSL2 X-ray sources and potential stellar catalog counterparts, where we corrected the position of the stellar 
counterpart for proper motion, if the proper motion is given in the catalog. This procedure includes optical and near-infrared (NIR) brightness cuts to limit the number of chance alignments with faint sources that are unlikely to be responsible for Xray emission. The appropriate magnitude cuts depend on the Xray detection limit, i.e., the conversion between observed count rate and flux, and the expected ratio between X-ray and optical or NIR fluxes. We then tested our procedure against random sources, motivate the used matching distances, and discuss the further applied selection procedures; a flow chart of our matching procedure is given in Appendix A.

\subsection{X-ray fluxes}

The conversion between the measured count rates and the derived X-ray fluxes depends on the spectral model assumed for the X-ray source. Therefore, we did not adopt the X-ray fluxes given in the XMMSL2 catalog, which are estimated by applying a spectral model typical for AGN; instead, we used our own conversion by adopting a spectral model that is appropriate for stellar X-ray sources. Specifically, we assumed optically thin emission and adopted an APEC thermal plasma model with a temperature of $5 \times 10^{6} \mathrm{~K}$ and solar metallicity. We neglected interstellar extinction because we expected to find most of the stellar counterparts within $150 \mathrm{pc}$. However, a few sources located in star forming regions might be affected by the interstellar absorption, but with the data at hand we cannot identify these sources. Furthermore, we converted these fluxes into the ROSAT band to compare the XMMSL2 X-ray fluxes with previous measurements and used a count rate [cts $\mathrm{s}^{-1}$ ] to flux conversion factor of $1.24 \times 10^{-12} \mathrm{erg} \mathrm{cm}^{-2} \mathrm{~s}^{-1}$ for the soft and total band. The conversion factor is relatively insensitive to the assumed temperature; for the $2-35 \times 10^{6} \mathrm{~K}$ range, it changes by $6 \%$ and $15 \%$ for the total and soft band, respectively. The hard band is generally less suitable to observe stellar X-ray sources because coronal X-ray sources are typically rather soft X-ray emitters, unless they are heavily absorbed and the effective area of the $X M M$ Newton decreases for high energy photons. We formally adopted a flux conversion factor of $5.93 \times 10^{-10} \mathrm{erg} \mathrm{cm}^{-2} \mathrm{~s}^{-1}$ per count rate of $1 \mathrm{cts} \mathrm{s}^{-1}$ for the hard band, but this value is - naturally very sensitive to the assumed model temperature, and we do not expect stellar sources to be detected only in the hard band.

The detection limit of the XMMSL2 catalog is typically $\sim 0.4 \mathrm{cts} \mathrm{s}^{-1}$ for a source passing through the center of the detector at a typical background level. With the adopted conversion factor this corresponds to an X-ray flux of $5 \times 10^{-13} \mathrm{erg} \mathrm{cm}^{-2} \mathrm{~s}^{-1}$, which we used to derive the minimum optical brightness of potential stellar counterparts.

\subsection{Minimal optical brightness of the stellar XMMSL2 sources and magnitude cutoff}

With our estimate of the limiting XMMSL2 flux and the saturation limit of stellar X-ray emission, we can compute the minimal bolometric flux of a possible stellar counterpart to an XMMSL2 X-ray source. Given this minimal bolometric flux, we used Table 3 of Worthey \& Lee (2011) to calculate the minimum optical brightness in different photometric bands as a function of the effective temperature (cf. Sect. 3.6), again neglecting interstellar absorption, and show the computed magnitudes in the $V, G$, and $J$ bands versus the effective temperature in Fig. 1. As is clear from Fig. 1, all stellar XMMSL2 sources ought to be relatively bright in the optical with $G \lesssim 14$ mag. Furthermore, the stellar sources are also bright in the infrared band with

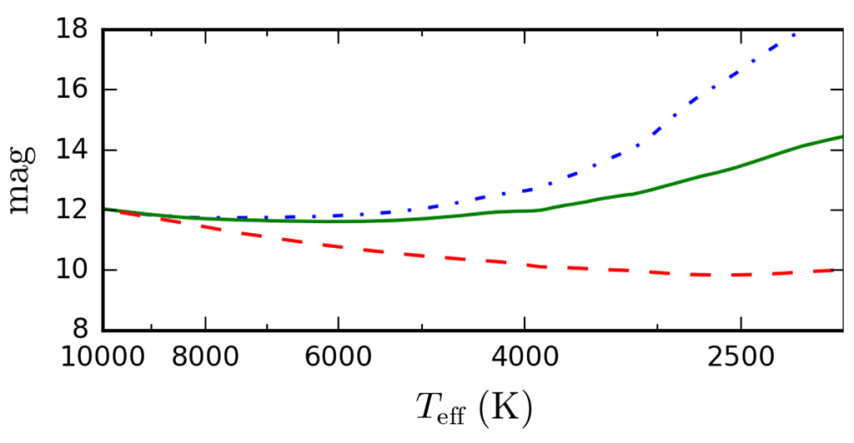

Fig. 1. Apparent magnitude in different photometric bands for a star with saturated X-ray emission at the detection limit of the XMMSL2 catalog vs effective temperature. The blue dash-dotted line indicates the $V$ band; the green solid line indicates the $G$ band; and the red dashed line indicates the $J$ band.

$J \lesssim 12 \mathrm{mag}$; in particular, very late spectral type dwarfs can be faint in the $V$ and $G$ bands, but should still be bright in the $J$ band of 2MASS.

In practice, the magnitude limit shown in Fig. 1 is not sharp. First of all, there appears to be some intrinsic scatter in the saturation limit (Pizzolato et al. 2003) and, second, sources might be caught during an X-ray flare during the slew survey observations and therefore produce more X-ray flux than expected. Hence, the $L_{\mathrm{X}} / L_{\text {bol }}$ ratio of coronal sources detected in the XMMSL2 could be higher than the saturation limit and their optical brightness lower than the predictions shown in Fig. 1. For example, Stelzer et al. (2006) reported a flare with a peak X-ray luminosity 200-300 times above the quiescence emission and with an increase in optical brightness of $\Delta V=6 \mathrm{mag}$ for the star LP 412-31; however, these extreme flare events are rare and it is unlikely that XMM-Newton slews over a star during the peak of such an extreme flare.

Yet to allow for some margin in these cases, we adopted a magnitude cutoff at $G=16 \mathrm{mag}$ for Gaia sources, at $J=12 \mathrm{mag}$ for 2MASS sources and no cutoff for Tycho 2 sources; however, if neither a Tycho 2 nor a 2MASS counterpart is found, the Gaia cutoff is set to $G=15 \mathrm{mag}$. These cutoff values are clearly sufficient to find all potential stellar counterparts emitting at the $\mathrm{X}$-ray saturation level. The achieved stellar activity margin depends on spectral type, for example, for stars with an effective temperature of $3000 \mathrm{~K}$ (spT: M5V) the X-ray flux limit is about $\log \left(F_{\mathrm{X}} / F_{\text {bol }}\right)<-2.2$ at minimum optical brightness. We remark in passing that the adopted magnitude cutoffs are well above the completeness limits of the Gaia and 2MASS catalog, such that all stellar XMMSL2 sources ought to be included in the catalogs.

\subsection{Random matches and magnitude distribution}

With its 1.1 billion sources the mean distance between two Gaia entries is about 20 arcsec, thus finding a Gaia catalog entry in the vicinity of an XMMSL2 source is not surprising. In order to investigate the influence of random coincidences on our matching procedures, we carried out Gaia identifications with randomly generated X-ray sources. Since both the XMMSL2 catalog and the stellar catalogs chosen for matching are nonuniform, it is important to preserve the global spatial distribution of the Xray sources in the randomly generated X-ray samples; this is achieved by using all cataloged XMMSL2 sources, but shifting their positions uniformly between a distance of 240 arcsec and 1200 arcsec along a randomly chosen direction. 


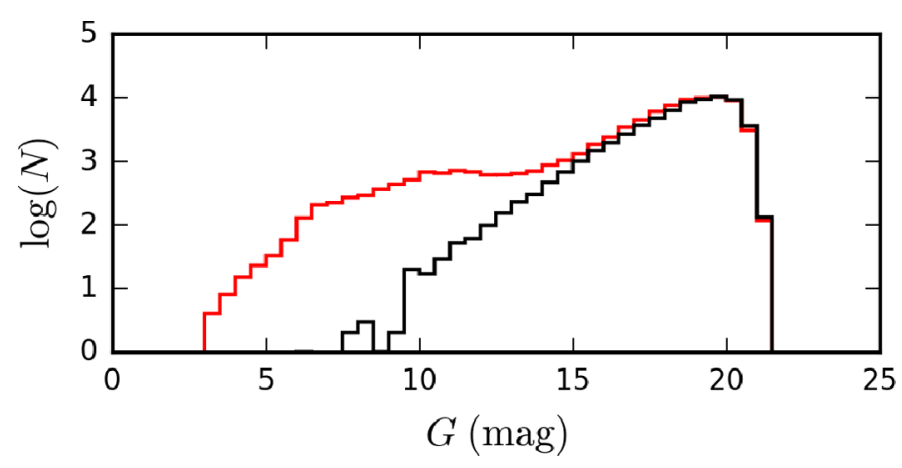

Fig. 2. Magnitude distribution of the Gaia counterparts. The red line indicates the magnitude distribution of the Gaia associations of the real XMMSL2 sources; and the black line indicates the magnitude distribution of the Gaia associations with the randomly generated sources

In Fig. 2 we show the thus obtained $G$ magnitude distribution of all Gaia matches (using a matching distance of 20 arcsec to the XMMSL2 sources) as well as that of the randomly generated XMMSL2 sources. Obviously, these distributions substantially differ from each other. While the Gaia magnitude distribution of the true XMMSL2 sources is bimodal with a broad first peak near $G \approx 10 \mathrm{mag}$, the Gaia magnitude distribution of the random XMMSL2 sources steadily increases up to the magnitude cutoff of the Gaia catalog near $G \approx 20$ mag. Interestingly, the identified number of true XMMSL2 sources exceeds that of randomly generated XMMSL2 sources up to $G \approx 20 \mathrm{mag}$. Thus, even at faint magnitudes some of the Gaia counterparts appear to be the correct, albeit not necessarily stellar, identifications. However, it is also clear that for a magnitude $G \approx 14 \mathrm{mag}$, the chance to obtain a random match exceeds $50 \%$ using the matching distance of 20 arcsec.

\subsection{Choice of the matching distance}

Next, we considered the (differential) number of matched XMMSL2-Gaia sources (choosing only Gaia entries with $G<15 \mathrm{mag}$ ) as a function of matching distance and show the resulting histograms for the real XMMSL2 sources and the randomly generated XMMSL2 sources in Fig. 3. Again, the two distributions differ substantially. The distribution of the randomly generated sources increases linearly as expected, while the distribution of the real XMMSL2 sources is bimodal. At small matching distances it is dominated by a Gaussian-type distribution up to a distance of 15 arcsec. We find this distribution to be better fitted by a double Gaussian than by a single Gaussian distribution for the XMMSL2 sources. However, this is only an empirical description without any deeper physical meaning. At larger distances the distribution of the real XMMSL2 sources approximates the linear distribution of the randomly generated sources. The peak at small distances contains the true matches, while the linearly increasing population of matches represents random associations. In Fig. 3 we also plot the corresponding distributions resulting from the 2MASS catalog using a magnitude limit of $J=12$ mag and the full Tycho 2 catalog. These distributions are qualitatively similar to the Gaia distribution and differ only quantitatively because of the smaller number of cata$\log$ entries.

\subsubsection{Matching probability}

The distribution of the real XMMSL2 sources can be well fitted with a double Gaussian (describing the uncertainty in the

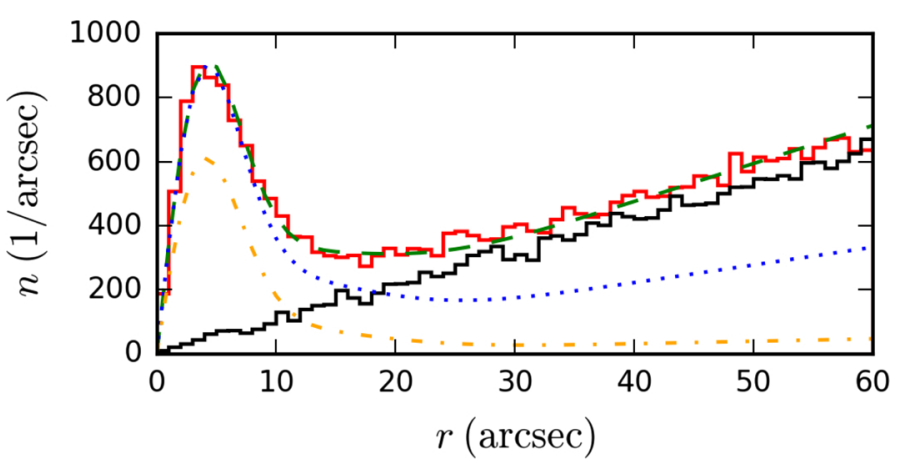

Fig. 3. Distribution of the distances between the XMMSL2 sources and the stellar counterparts. The red solid line represents a histogram of the distances between the XMMSL2 sources and the Gaia counterparts. The black solid line represents a histogram of the distances between the randomly generated sources and the Gaia counterparts. The dashed and dotted curves represent the best fits of Eq. (1) for the different catalogs with the parameters of Table 1 . The green dashed curve represents the Gaia catalog, the blue dotted curve represents the 2MASS catalog, and the orange dash-dotted curve represents the Tycho 2 catalog.

Table 1. Fitted parameters of Eq. (1).

\begin{tabular}{llll}
\hline \hline & Gaia & 2MASS & Tycho2 \\
\hline$A\left[1 / \operatorname{arcsec}^{2}\right]$ & $310 \pm 6$ & $318 \pm 6$ & $233 \pm 5$ \\
$B\left[1 / \operatorname{arcsec}^{2}\right]$ & $29 \pm 1$ & $27 \pm 1$ & $11.7 \pm 0.6$ \\
$M\left[1 / \operatorname{arcsec}^{2}\right]$ & $11.89 \pm 0.09$ & $5.54 \pm 0.06$ & $0.77 \pm 0.03$ \\
\hline
\end{tabular}

XMMSL2 positions) and a linear curve (describing the random matches) using the ansatz

$n(r)=A \cdot r \cdot \exp \left(-\frac{r^{2}}{2 \sigma_{1}^{2}}\right)+B \cdot r \cdot \exp \left(-\frac{r^{2}}{2 \sigma_{2}^{2}}\right)+M \cdot r$.

In Eq. (1) the parameters $\sigma_{1}$ and $\sigma_{2}$ are the standard deviations of the Gaussian distributions, which are independent of the matching catalog; we find $\sigma_{1}=4.0$ arcsec and $\sigma_{2}=9.9$ arcsec. The values for the parameters $A, B$, and $M$ depend on the source densities of the catalogs. In Table 1 we provide the best fit parameters for the catalogs used in this paper, and in Fig. 3 we give a visual representation of the best fit curves.

With the fitted parameters of Eq. (1) the probability $p$ of a match at the distance $r$ to be the true counterpart can be estimated through the expression

$p(r)=\frac{A \cdot r \cdot \exp \left(-\frac{r^{2}}{2 \sigma_{1}^{2}}\right)+B \cdot r \cdot \exp \left(-\frac{r^{2}}{2 \sigma_{2}^{2}}\right)}{A \cdot r \cdot \exp \left(-\frac{r^{2}}{2 \sigma_{1}^{2}}\right)+B \cdot r \cdot \exp \left(-\frac{r^{2}}{2 \sigma_{2}^{2}}\right)+M \cdot r}$.

Although the standard deviations $\sigma_{1}$ and $\sigma_{2}$ take the same value for all matching catalogs, the probability varies for a specific matching distance because for a catalog with a lower source density one is less likely to find a random association. The differences in the source densities are caused by the magnitude ranges covered by the various catalogs. However, the matching probability does not consider the different brightnesses within a cata$\log$. Therefore, the fraction of spurious identifications ought to be larger near the magnitude cutoff. 


\subsubsection{Missed and spurious identifications}

The number of stellar identifications of the XMMSL2 sources missed in our sample, i.e., the completeness, and the number of spurious identifications in the sample, i.e., the reliability, depend on the chosen matching criteria. Especially, the probability cutoff above which we assume matches to be the true counterpart controls these two characteristics (cf. Sect. 3.4.1). The number of spurious identifications $N_{\text {spurious }}$ in the sample with a constant matching distance $r$ can be estimated through

$N_{\text {spurious }}=\int_{0}^{r} M r^{\prime} \mathrm{d} r^{\prime}$.

In our sample the matching distance is not constant, but depends on the catalog used for the identification. The differential matching probability, defined in Eq. (2), gives the probability for a match at the distance $r$ to be the true counterpart. Hence we calculated the probabilities $p_{i}$ of each match up to a distance of 40 arcsec where the probability to be the true counterpart drops to below $1 \%$ for all our catalogs, i.e., these matches are negligible.

Given the probabilities $p_{i}$ and the chosen probability cutoff, the number of spuriously identified XMMSL2 sources can be estimated by summing the inverse probabilities of all sources with a probability higher than the cutoff $N_{>\text {cutoff }}$ For a XMMSL2 source with $N_{\text {matches }}$ matches, the probability that none of the matches is the true counterpart is given by the product of the inverse probabilities of all matches. Hence, we estimated the number of spuriously identified sources through

$N_{\text {spurious }}=\sum_{i}^{N_{>\text {cutoff }}} \prod_{j}^{N_{\text {matches }}, i}\left(1-p_{j}\right)$

and we defined the reliability $r$ of the matched sample as

$r=\frac{N_{>\text {cutoff }}-N_{\text {spurious }}}{N_{>\text {cutoff }}}$

We estimated the number of missed identifications by summing the probabilities of all matches with a probability lower than the cutoff $N_{<\text {cutoff }}$

$N_{\text {missed }}=\sum_{i}^{N_{\text {cuutoff }}} p_{i}$

and define the completeness $C$ as

$C=\frac{N_{>\text {cutoff }}}{N_{>\text {cutoff }}+N_{\text {missed }}}$

\subsection{Associations in multiple catalogs}

A few XMMSL2 sources have counterparts in several catalogs, therefore we must determine whether we are considering the same counterpart or not. In some catalogs the identifications of other catalogs are specified; for example, the TGAS sample of the Gaia DR1 contains the Tycho2 identifier, which is, in this specific case, based exclusively on apparent sky distance. If no identifier is specified, we associated counterparts if their distance is smaller than 1 arcsec or their extrapolated $V$ band magnitude difference is smaller than $1.5 \mathrm{mag}$ and their distance is smaller than 4 arcsec. The exact distances do not influence the result significantly. We chose the closest match if these conditions are met by multiple counterparts. For measurements given in multiple catalogs, we used the value of the catalog with the highest accuracy of the respective measurement. To estimate the probability of a random match, we used the catalog with the highest probability.

\subsection{Additional stellar properties}

With the magnitudes in the available photometric bands, we estimated the effective temperatures, the bolometric magnitudes and fluxes and the $V$ band magnitudes for the sources with a MASS and Gaia counterpart only. We adopted the relations in Table 3 of Worthey \& Lee (2011), applying solar metallicity and a surface gravity of $g=10^{4.5} \mathrm{~cm} \mathrm{~s}^{-2}$ corresponding to dwarfs and used a linear interpolation of the values given in the table. We did not perform any corrections for extinction. Since Worthey \& Lee (2011) provided the colors in the photometric system of Bessel \& Brett, we applied the relation given by Carpenter (2001) and in Explanatory Supplement to the 2MASS All Sky Data Release and Extended Mission Products ${ }^{1}$ to obtain the 2MASS colors. We further adopted the correlation to the Gaia band from Jordi et al. (2010) and the Gaia Data Release Documentation ${ }^{2}$.

For the calculation we adopted the $V-K$ color for the sources with a counterpart in the Tycho 2 catalog and the 2 MASS catalog, while we used the $B-V$ color and the $J-K$ color for the sources with a counterpart only in the Tycho 2 catalog or the 2MASS catalog, respectively. We could not estimate the bolometric flux and the effective temperature for a few sources $(\sim 2 \%)$ because they have a Gaia counterpart only or they are extremely red and lie outside the region defined in Table 3 of Worthey \& Lee (2011).

We find a trigonometric parallax for $57 \%$ of the stellar XMMSL2 sources and for these sources, we further estimate the distance, the $V$ band and bolometric absolute magnitude, and the bolometric and the X-ray luminosity.

\subsection{Cleaning procedures}

In our catalog we introduce different XMMSL2 source flags, if the sources have measurements of low quality or to indicate likely nonstellar objects. A few object classes are identified as stellar sources by the procedures outlined above, which are not the focus of this work; examples are high- and low mass X-ray binaries, where the X-ray emission is not predominantly produced by the star, but by matter accreted onto a compact object. Many of these objects were already excluded because they generally have faint optical counterparts due to their very large X-ray/optical flux ratios, but we additionally excluded sources that have a known accreting object in the SIMBAD database (Wenger et al. 2000) within a distance of $30 \operatorname{arcsec}$ to the XMMSL2 detection. Furthermore, we flagged sources that have a known galaxy cluster within 60 arcsec or an AGN located within 30 arcsec as listed the SIMBAD database. For such X-ray sources, the stellar object and extragalactic object are both plausible counterparts, given the available information.

We additionally flagged all sources that are not detected in all 2MASS bands and that have no association in other catalogs. Furthermore, the stellar identifications are unreliable if they are flagged as extended in the 2MASS catalog or if they are

\footnotetext{
1 http://www.ipac.caltech.edu/2mass/releases/allsky/ doc/explsup.html

2 https://gaia.esac.esa.int/documentation/GDR1/
} 


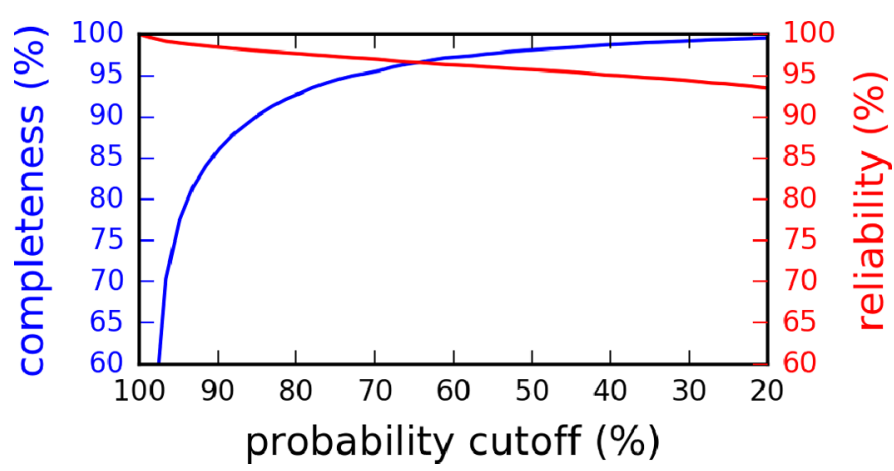

Fig. 4. Completeness (blue curve) and reliability (red curve) of the sample as a function of the probability cutoff.

detected in the XMMSL2 hard band only. We expected sources to be affected by optical loading (i.e., X-ray events created by an excess of optical photons in the pn camera) if they are flagged in the XMMSL2 catalog and do not have a RASS counterpart (cf. Sect. 5). Some sources have erroneous 2MASS photometry or an unusual color and very likely the derived magnitudes and stellar properties are unreliable. Additionally, we flagged sources with a high $\mathrm{X}$-ray to the bolometric flux ratio. Specifically, we flagged sources with a high $F_{\mathrm{X}} / F_{\text {bol }}$ ratio only in the slew survey and set an additional flag for sources that consistently have a high $F_{\mathrm{X}} / F_{\text {bol }}$ ratio in both the slew and the RASS surveys and another flag is used for sources without RASS counterpart.

All used flags are summarized in Appendices A and B. We generally excluded flagged sources from our subsequent analysis, but discuss some of their properties in the following sections.

\section{Results}

\subsection{Stellar counterparts, completeness, and reliability}

Figure 4 shows the completeness and reliability of the sample and its dependence on the matching probability for the nonflagged sources. To balance completeness and reliability, we choose a matching probability $>2 / 3$, i.e., about intersection of the two curves, to derive the stellar catalog of XMMSL2 sources and obtain a completeness of $96.3 \%$ (Eq. (6)) and a reliability of $96.7 \%$ (Eq. (4)) ignoring sources that are flagged by our cleaning procedure in the calculations. The matching radii correspond to 10.8 arcsec, 13.9 arcsec, and 19.9 arcsec for sources with a counterpart in the Gaia, 2MASS and Tycho2 catalogs, respectively.

Combing these matching radii with our adopted magnitude cutoffs, we find at least one stellar counterpart for 6815 of the 23252 XMMSL2 sources. Our cleaning procedures reduce the sample to 5920 sources, implying that $25.5 \%$ of the XMMSL2 sources are stellar sources.

\subsection{Single and multiple counterparts}

We specify the number of selected stellar counterparts per XMMSL2 source in Table 2. For the 5042 XMMSL2 sources with a single counterpart we present the histogram of the distances between the XMMSL2 sources and the counterparts in Fig. 5. No strong discontinuities are visible at the adopted matching distances, only at $\approx 14$ arcsec, i.e., for 2 MASS identification without a Tycho2 counterpart, a slight drop is visible. Sources with a distance $>$ arcsec have a Tycho 2 identification

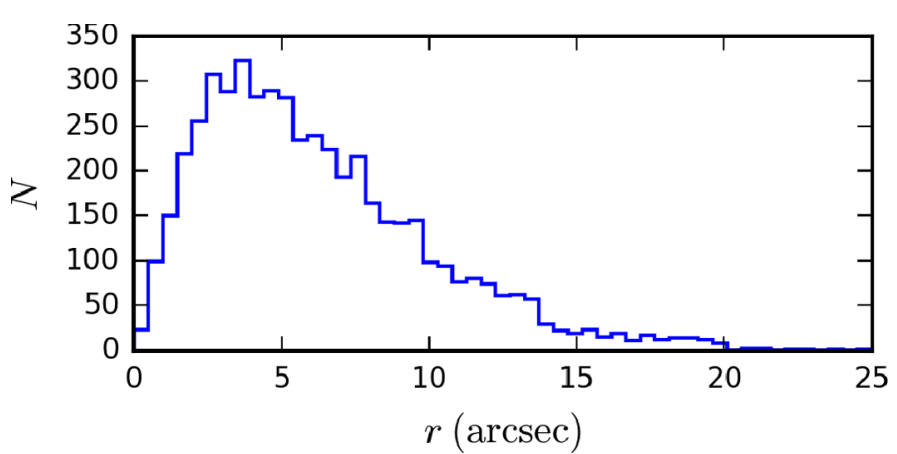

Fig. 5. Distances between the XMMSL2 sources and the counterparts for the 5042 XMMSL2 sources with a single match.

Table 2. Number of multiple counterparts.

\begin{tabular}{cc}
\hline \hline Number of stellar counterparts & Number of sources \\
\hline 1 & 5042 \\
2 & 761 \\
3 & 103 \\
$>3$ & 14 \\
\hline
\end{tabular}

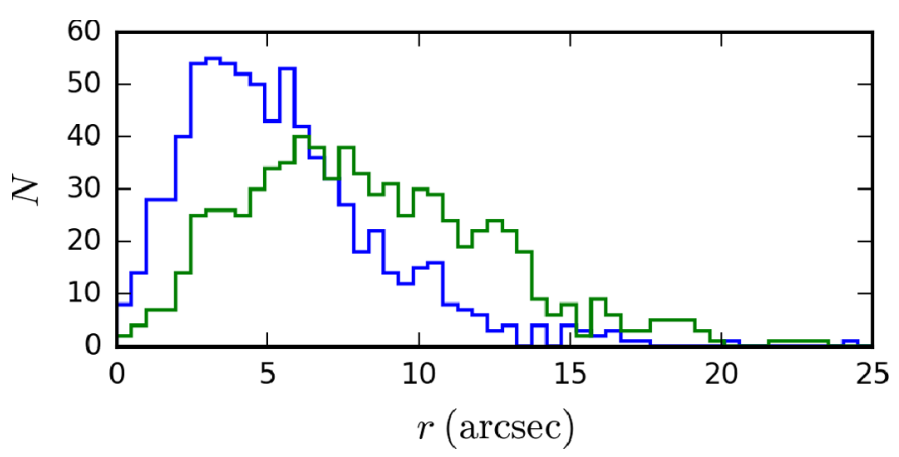

Fig. 6. Distances between the XMMSL2 sources and the counterparts for the 761 XMMSL2 sources with two matches. The blue curve shows the distance of the sources with the higher matching probability; the green curve of the sources with the lower matching probability.

and the few sources at a distance larger than 20 arcsec have a counterpart in the BrightStar or Lepine catalog that we include because of the small source density of these catalogs.

We find that roughly $15 \%$ of the stellar XMMSL2 sources have more than one plausible stellar counterpart. In Fig. 6 we show the distances between the XMMSL2 sources and the counterparts for the 761 XMMSL2 sources with two matches. The mean angular separation of the counterparts with the higher matching probability for each XMMSL2 sources is smaller, but overall the two distributions are quite similar, i.e., both distributions have a maximum at small distances and the number of sources decreases for larger distances. In Fig. 7 we show the histogram of the angle between the XMMSL2 source and the counterparts for the sources with two counterparts. The distribution has a maximum at $180^{\circ}$, which implies that the XMMSL2 source tends to lie between both candidates. Thus, the cataloged XMMSL2 source is often likely to be a combination of the Xray emissions of the two sources. We ignore sources with multiple counterparts when investigating the properties of the stellar counterparts. 


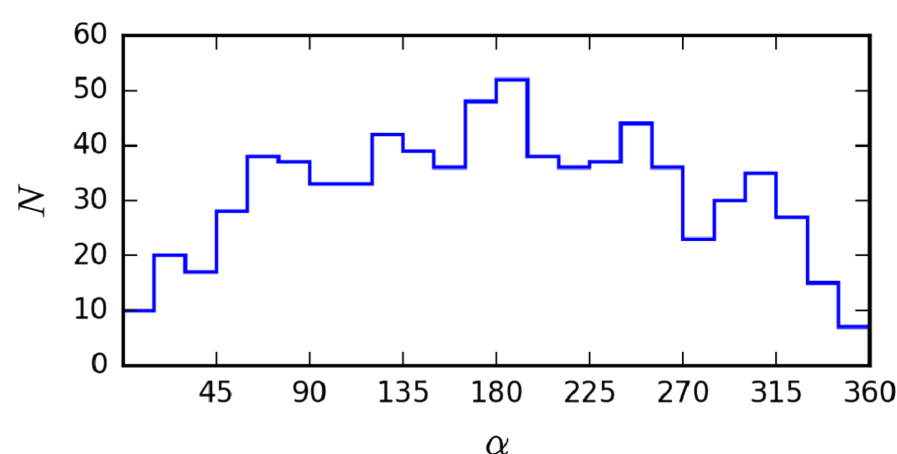

Fig. 7. Distribution of the angle between the XMMSL2 source and the counterpart for the 761 sources with two counterparts.

Table 3. Catalogs of the counterparts.

\begin{tabular}{lccc}
\hline \hline Catalog & $N$ & Catalog & $N$ \\
\hline TMG & 3595 & $\mathrm{M}$ & 116 \\
MG & 1784 & $\mathrm{~T}$ & 54 \\
TM & 318 & $\mathrm{G}$ & 15 \\
TG & 36 & other & 2 \\
\hline
\end{tabular}

Notes. G: Gaia, M: 2MASS, T: Tycho2.

\subsection{Catalogs of the counterparts}

In Table 3 we denote the catalogs providing of the most probable counterpart. Most counterparts have a Gaia and 2MASS identification as expected with 490 counterparts missing in the Gaia DR1 catalog. This is likely caused by the known incompleteness of the Gaia DR1 catalog. Furthermore, some of the Gaia sources cannot be identified with their 2MASS counterparts, possibly because of observing epochs that differ by about 15 years and unknown proper motion; 107 counterparts do not have a 2MASS counterpart. Multiple stars that are resolved in the Tycho2 or Gaia catalog, but not in 2MASS, could explain most of the 107 sources that do not have a 2MASS counterpart. The two sources denoted as "other" in Table 3 only have a counterpart in the Lepine or BrightStar catalog with extremely high proper motions $\left(>4 \operatorname{arcsec} \mathrm{yr}^{-1}\right)$ and therefore, the 2MASS and Gaia counterparts, having no proper motion, lie outside of our initial matching radius of 40 arcsec (cf. Appendix A).

\subsection{Comparison to the identifications of Saxton et al. (2008)}

The XMMSL2 catalog provides identifications and classifications for about $70 \%$ of the X-ray sources as a result of a crossmatch with the SIMBAD, NED, and other databases and catalogs. However for some sources the classification only contains the region of the electromagnetic spectrum in which the source has been detected, for example, "X-ray", and provides little or no insight into the physical nature of the source. The classification adopted in the XMMSL2 catalog Saxton et al. (2008) uses different catalog resources and enables an independent comparison with our results.

There is large overlap in the identification with 4231 sources consistently classified as stellar; the XMMSL2 catalog identifies a total of 5094 sources as stars. Our stellar identification was not confirmed for 1689 sources, however the vast majority (1671) of these have either no identification or the classification contains only the region of the electromagnetic spectrum. The XMMSL2 contradicts our stellar identification only for 18

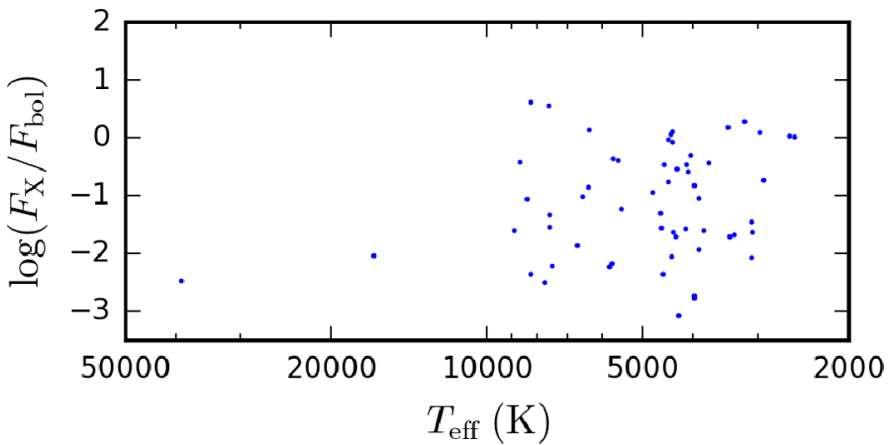

Fig. 8. $F_{\mathrm{X}} / F_{\text {bol }}$ distribution as a function of effective temperature of the sources classified as stellar in the XMMSL2 catalog, but missing a stellar counterpart with our approach.

$(0.4 \%)$ sources, typically referring to an additional plausible counterpart.

On the other hand, 863 sources are classified as stars in the XMMSL2 catalog, in which we did not find a stellar counterpart satisfying our selection criteria. Out of these, 531 were excluded by our cleaning procedures, which argues against a true stellar identification. To validate the reliability of the remaining 332 stellar identifications, we inspected the $\log F_{\mathrm{X}} / F_{\text {bol }}$ ratio as a function of the effective temperature for the 59 sources for which colors from the SIMBAD database are available (see Fig. 8). For most of these sources, we find $\log F_{\mathrm{X}} / F_{\text {bol }}>-2$ or $T_{\text {eff }}>10000 \mathrm{~K}$ and $\log F_{\mathrm{X}} / F_{\text {bol }}>-3$, i.e., highly unlikely values for stellar X-ray sources. Overall, only a few of these sources remain as plausible stellar counterparts that are missed due to our chosen magnitude cutoff. Based on this fraction, we estimate that about $1 \%$ of the stellar counterparts are missed by our procedure due to the applied brightness limit.

\subsection{Validation of our procedure}

\subsubsection{Application of our procedure to the EMSS catalog}

We validated the reliability of our identification approach by applying our procedures to the Extended Medium-Sensitive Survey (EMSS; Gioia et al. 1990; Stocke et al. 1991). The EMSS catalog contains 835 X-ray sources detected in 1435 pointings with the Imaging Proportional Counter (IPC) on board the Einstein Observatory at high galactic latitudes $\left(|b|>20^{\circ}\right)$. More than $96 \%$ of the EMSS sources have been individually identified with spectroscopically classified counterparts. The reliability of these identifications should be very high, therefore the EMSS catalog is an ideal tool to test the reliability of our automatic procedure.

To identify the stellar content of the EMSS, we used basically the same procedure as for the XMMSL2 catalog, but we find a single Gaussian plus linear curve to describe adequately the matching distance distribution of the EMSS sources. Furthermore, we searched in the SIMBAD database for accreting and extragalactic objects within 50 arcsec, which is the typical $90 \%$ confidence error circle radius of the EMSS sources.

We identified 210 of the 835 EMSS sources as stellar, whereas we expected about 15 identifications to be spurious and a similar number to be missed by the probability cutoff. These numbers can be compared to the identifications of the EMSS catalog, which contain 217 stars; 192 sources are consistently classified by the EMSS catalog and by our procedure.

The EMSS catalog thus identifies 25 sources as stars that we did not find with our procedure as stellar sources. One of 


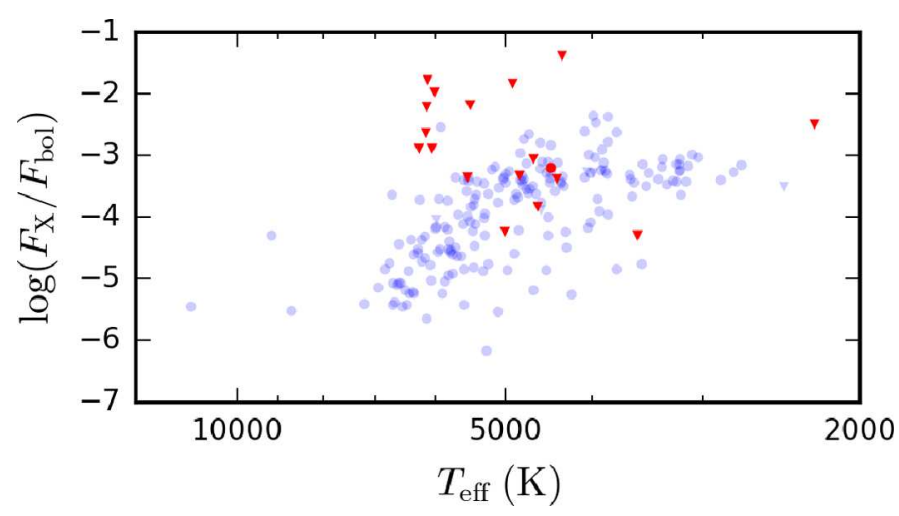

Fig. 9. $F_{\mathrm{X}} / F_{\text {bol }}$ distribution of the EMSS sources as a function of effective temperature. The blue symbols represent sources consistently identified as stars, and red symbols show sources classified as stars by our procedure, but with contradicting EMSS classification. The triangles denote possible extragalactic sources.

these sources is marked as a white dwarf and 7 are marked as cataclysmic variables. Hence, 17 sources are stellar X-ray sources that are missed by our procedure, 10 because of the large distance to the EMSS source, 6 because they have magnitudes of $J>12 \mathrm{mag}$, and one case is uncertain. We applied the same magnitude cutoff to the EMSS catalog as for the XMMSL2 catalog, which is a simplification and not an optimal cutoff for all EMSS sources. Hence, slightly fewer sources are missed because of the probability cutoff than expected.

For 18 sources the EMSS classification explicitly contradicts our stellar identification. However, for most of these sources there are two plausible counterparts in the SIMBAD database, i.e., one stellar counterpart and one extragalactic counterpart. So the stellar identifications may be random associations, which is supported by the number of flagged sources in the total sample (23/210) compared to consistently identified stars (6/192). The number of 18 random associations is comparable to the expectation of 15 spurious identifications.

In Fig. 9 we present the $\log F_{\mathrm{X}} / F_{\text {bol }}$ distribution of the 210 EMSS sources that we identify as stellar sources. Obviously, about half of the sources that have a contradicting classification in the EMSS catalog lie above the distribution of the sources that are consistently classified as stars. The other are at least plausible stellar X-ray sources, but we find that they have generally a high angular separation and further they are mostly flagged as having a plausible extragalactic counterpart. Therefore, there is a high chance that these stellar counterparts are random associations.

Yet, in summary the completeness and reliability of our automatic identifications reach the expectations. Therefore we are confident that the reliability and completeness calculated in Sect. 3.4.2 represent the true reliability and completeness of the stellar XMMSL2 sample.

\subsubsection{Matching with the Chandra Source Catalog}

In comparison to XMM-Newton, the Chandra X-ray Observatory (CXO; Weisskopf et al. 2000, 2002) provides more accurate positions of the X-ray sources owing to its sub-arcsec on-axis point spread function (PSF). However, in its current release 1.1 the Chandra Source Catalog (CSC; Evans et al. 2010) covers less than $1 \%$ of the sky. Nevertheless, the precise positions of the CSC sources give us the opportunity to validate some of our stellar identifications. Therefore, we performed a crossmatch of our stellar identifications with the CSC, applying a matching distance of $60 \mathrm{arcsec}$. Although the CSC sources

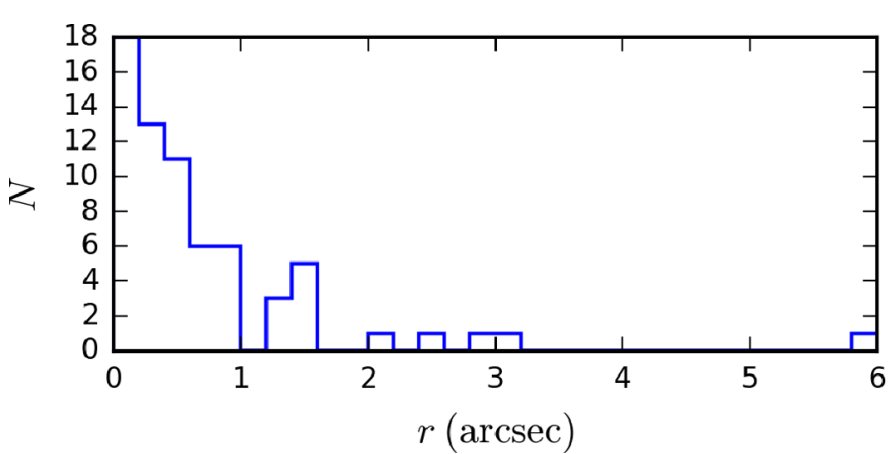

Fig. 10. Angular separation between the stellar identification and the closest CSC counterpart for the XMMSL2 sources with a single match.

generally have highly accurate positions, for some sources the positional uncertainty is much larger, and hence, we excluded sources with a $95 \%$ confidence error circle radius larger than 5 arcsec from our analysis.

In this fashion we find a CSC counterpart for 94 of the 5920 stellar identifications and show the angular separation between the stellar source and the CSC counterpart for the XMMSL2 sources with a single stellar identification in Fig. 10. For most of the sources (86) the distance between the CSC source and our best stellar identification is $<2$ arcsec as expected for the CSC sources or a larger angular separation can be explained by an unusual high positional uncertainty of the CSC source. For four sources the position of the CSC source indicates that our second best stellar counterpart is the correct identification of the X-ray source, and in four cases none of our stellar identifications lie within the $95 \%$ confidence error circle of the CSC source.

In summary, the crossmatch with the CSC confirms at least one of our stellar sources for $95.7 \%$ of the XMMSL2 sources, which is in good agreement with the reliability calculated in Sect. 3.4.2.

\subsection{Catalog release}

We released the catalog of the stellar XMMSL2 sources at VizieR. This catalog contains our stellar identifications with a matching probability $>2 / 3$. While we discuss in this paper only the properties of the unflagged sources, the released catalog also includes the stellar counterparts that are flagged by our cleaning procedure. The XMMSL2 sources with multiple stellar counterparts have multiple entries in our catalog, one entry for each counterpart. We describe all new columns of our catalog in Appendix B.

\section{RASS counterparts}

Before turning to the physical properties of our stellar counterparts, we compared and crossmatched the results from the XMMSL2 to the second RASS-catalog. Since the RASS flux limit is deeper $\left(\sim 2 \times 10^{-13} \mathrm{erg} \mathrm{cm}^{-2} \mathrm{~s}^{-1}\right)$ than that of the $X M M-N e w t o n$ slew survey $\left(\sim 5 \times 10^{-13} \mathrm{erg} \mathrm{cm}^{-2} \mathrm{~s}^{-1}\right)$, one would naively expect that all XMMSL2 sources should have RASS counterparts. However, we found a RASS counterpart within 60 arcsec for only $75.2 \%$ of the sources; the matching fraction increases for multiple detected XMMSL2 sources to $91.6 \%$.

To investigate the properties of the stellar XMMSL2 sources without a RASS identification, we compared their distribution as a function of the apparent bolometric magnitude and X-ray activity level to the full sample. As shown in Fig. 11, the fraction of 

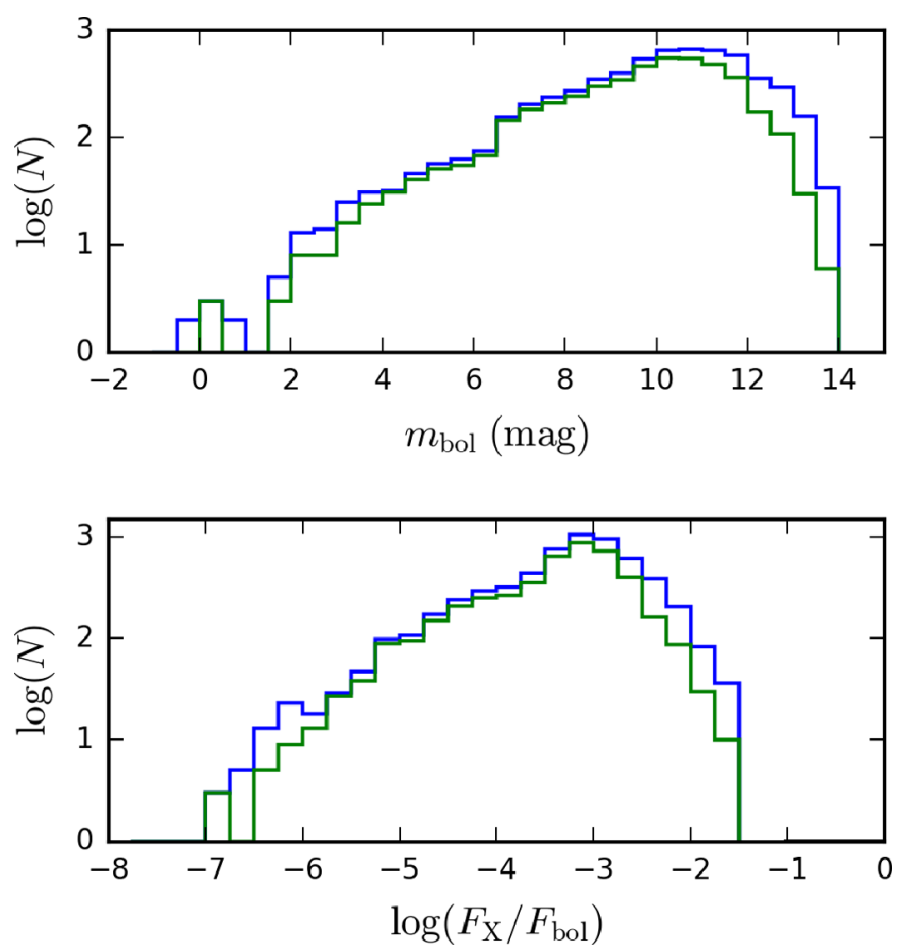

Fig. 11. Number of all stellar XMMSL2 sources (blue) and of those with a RASS counterpart (green) as a function of (top) apparent bolometric magnitude and (bottom) X-ray activity.

sources with a RASS identification are reduced for two different types of sources: first for bright sources and second for highly active sources. For high activity stars we expect frequent flaring; thus, the small fraction of RASS identification is caused by the intrinsic variability of these stars.

At bright magnitudes we expected the small fraction of RASS identifications to be due to optical loading; 102 of the stellar XMMSL2 sources are flagged in the XMMSL2 catalog as possibly affected by optical loading. However, 68 of these sources have a RASS identification and, hence, should be considered to be true X-ray emitters; RASS data are not affected by optical contamination. Furthermore, 6 sources brighter than 5 mag without a RASS counterpart are not flagged. Therefore we conclude that the influence of optical loading cannot be reliably determined with the slew data only and hence, the optical loading flag should be used as an indicator together with additional information about the source. The absolute number of XMMSL2 sources affected by optical loading is small and, therefore, intrinsic variability is the main reason why many stellar XMMSL2 sources do not have a RASS identification. We flagged those stellar XMMSL2 sources that are marked as possibly affected by optical loading and that do not have a RASS identification (cf. Sect. 3.7).

For the XMMSL2 sources with a RASS counterpart two independent X-ray flux measurements are available. We converted the measured count rates of the XMMSL2 and RASS sources by applying the conversion factors defined in Sect. 3.1 and by Schmitt et al. (1995), respectively. In Fig. 12 we compare the Xray fluxes measured by XMM-Newton and ROSAT for the stellar XMMSL2 sources. For most sources the XMMSL2 flux is higher than the RASS flux and the median flux ratio is 1.4. The difference generally increases with increasing X-ray activity of the star as indicated by the color coding of Fig. 12. For many XMMSL2 sources only 4-5 X-ray counts have been detected

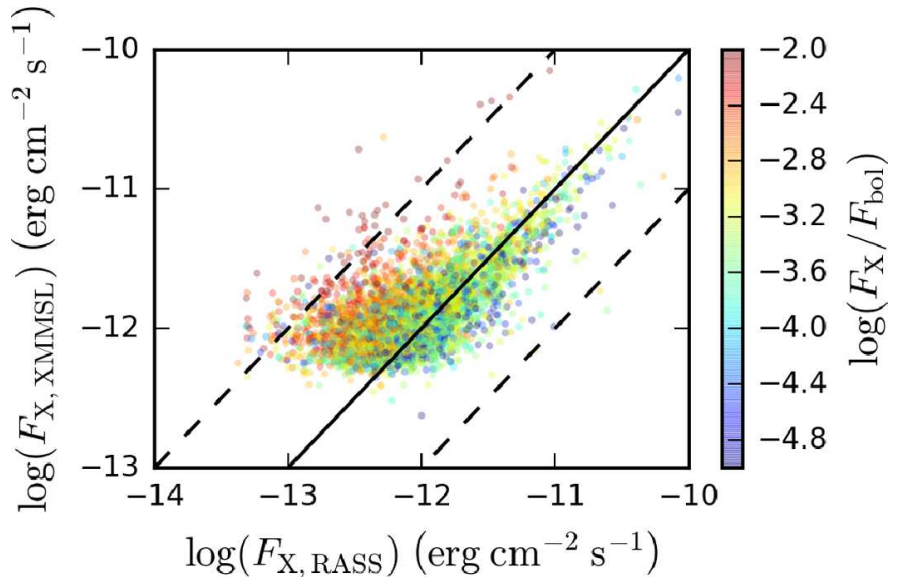

Fig. 12. Comparison of the X-ray fluxes measured by XMM-Newton and ROSAT. Solid line indicates the same X-ray flux in the XMMSL2 and RASS catalogs, dashed lines indicate flux difference of a factor of ten. The color scales with the stellar X-ray activity measured by $X M M$ Newton.

during the slew passages and therefore, the uncertainty of the $\mathrm{X}$-ray flux is very high, although the detection itself is significant. This causes a rather strong bias for the flux level of sources at the detection limit because many more sources lie just below the detection threshold than just above. Statistical fluctuations shift many of these above the threshold while the number of sources that have nominal fluxes above threshold, but remain undetected due to fluctuations to lower count numbers, is considerably smaller. In effect, we inevitably overestimate the average X-ray flux of sources close to the detection threshold. Additionally, differences in the conversion factors might induce the systematically higher flux of the XMM then of ROSAT. Furthermore, we assume that the larger deviations are caused by intrinsic variability generated by flares. The RASS flux is less affected by flares because the high flare fluxes have a smaller weight for RASS sources due to their longer exposure times. Additionally some sources can only be detected during a flare in the XMMSL2 catalog because the quiescent emission is below the XMMSL2 detection limit. In Fig. 12 the detection limit of the XMMSL2 catalog is visible at $\sim 5 \times 10^{-13} \mathrm{erg} \mathrm{cm}^{-2} \mathrm{~s}^{-1}$, while a detection limit of the RASS catalog is not noticeable because in the RASS catalog the exposure time, and hence, the detection limit is not constant over the sky but the exposure time varies between typically $400 \mathrm{~s}$ at the ecliptic equator and $\sim 40000 \mathrm{~s}$ at the poles.

\section{Properties of the stellar sample}

Next we discuss the X-ray properties of the crossmatched stellar XMMSL2 sources. We also address the nature of the identified stellar counterparts, where we restrict the discussion to unique identifications, i.e., sources with exactly one stellar counterpart. However, in Sect. 6.1 we use the stellar counterparts only to determine if the XMMSL2 source is stellar, and therefore, we also include sources with multiple counterparts.

\subsection{Number-flux-density distribution}

Figure 13 shows the derived number-flux-density distribution. At the X-ray bright end of the diagram, the number of sources decreases approximately linearly with increasing flux in the dou- 


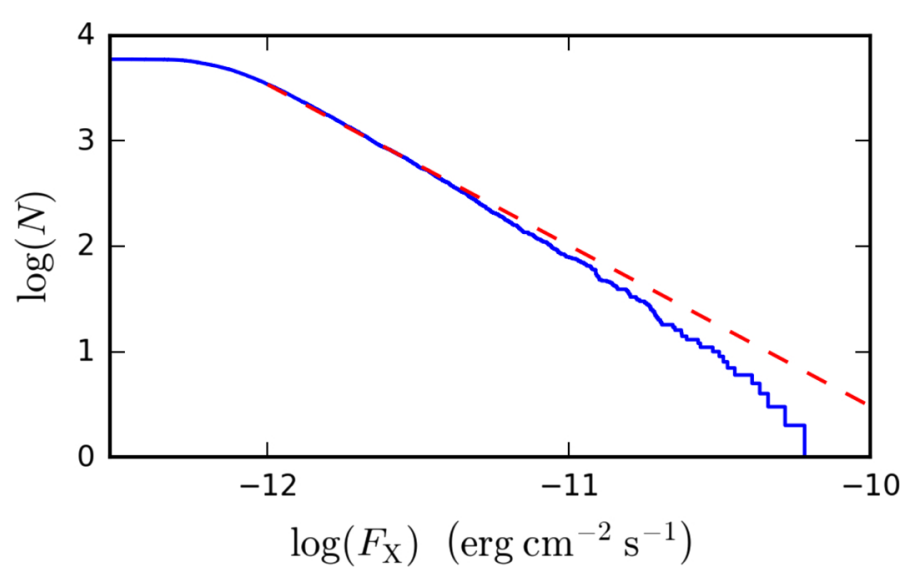

Fig. 13. Cumulative number-flux-density distribution of the stellar XMMSL2 sources. The red dashed line represents the best fit of the distribution.

ble logarithmic scale and we describe the distribution by the power law ansatz

$N(S)=k F_{\mathrm{X}}^{-\alpha}$,

where $N$ is the number of sources with a flux brighter than $F_{\mathrm{X}}, \alpha$ is the slope, and $k$ is the normalization. Applying the method of (Crawford et al. 1970), the best value of $\alpha$ can be estimated by maximizing the likelihood function

$L=M \ln (\alpha)-(\alpha+1) \cdot \sum_{i} \ln \left(\frac{F_{\mathrm{X}, i}}{F_{\mathrm{X}, \min }}\right)-M \ln \left(1-\left(\frac{F_{\mathrm{X}, \max }}{F_{\mathrm{X}, \min }}\right)^{-\alpha}\right)$,

where $M$ is the total number of sources, $F_{\mathrm{X} \text {,max }}$ and $F_{\mathrm{X} \text {,min }}$ are the brightest and faintest flux, respectively, and $F_{\mathrm{X}, i}$ is the flux of the $i$ th source.

The slope depends on the lower flux limit imposed on the sample when fitting the distribution because there is no hard detection limit due to different exposure times and background levels. Using only sources brighter than $F_{\mathrm{X}}=10^{-12} \mathrm{erg} \mathrm{cm}^{-2} \mathrm{~s}^{-1}$, we obtain a best value of $\alpha=1.53 \pm 0.03$, which agrees to a spatially uniform distribution.

The best fit differs from the distribution of the brightest sources that show a slightly steeper slope. However, these sources have a smaller weight and higher uncertainty due to their small numbers. Most sources are found at faint fluxes (see the logarithmic scale in Fig. 13), where a slope of $\alpha=1.53$ fits the distribution well.

\subsection{X-ray luminosities}

In Fig. 14 we present the distribution of the X-ray luminosities as a function optical color and compare the luminosities of our sample with those of the volume-limited NEXXUS sample (Schmitt \& Liefke 2004). The dwarf stars in our sample are up to two orders of magnitude brighter in X-rays than the most active NEXXUS sources of the same spectral type. Even the faintest sources in our sample are more luminous than the Sun, which is found at $\sim 3 \times 10^{26} \mathrm{erg} \mathrm{s}^{-1}$ and $\sim 5 \times 10^{27} \mathrm{erg} \mathrm{s}^{-1}$ at solar minimum and maximum, respectively (Peres et al. 2000). Hence, we only see the high luminosity tail of the stellar luminosity distribution in our sample. In addition, we expect many sources to be detected during a flare, which further biases our sample to high $\mathrm{X}$-ray luminosities.

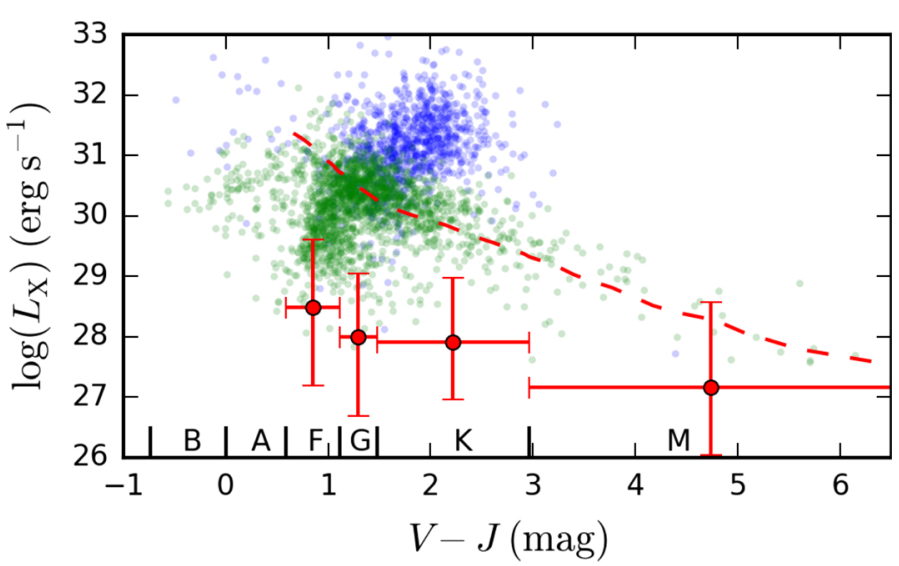

Fig. 14. X-ray luminosity distribution of the stellar XMMSL2 sources with known parallax. Main sequence stars and giants are shown as green and blue dots, respectively. The red crosses show the X-ray luminosities of the volume limited NEXXUS sample (Schmitt \& Liefke 2004). The extension in $x$-direction corresponds to the width of the spectral type, while the extension in $y$-direction shows the luminosity range in which $90 \%$ of the NEXXUS dwarfs of the specific spectral type lie. The red dashed line represents the saturation limit at $L_{\mathrm{X}} / L_{\mathrm{bol}}=10^{-3}$ for dwarfs.

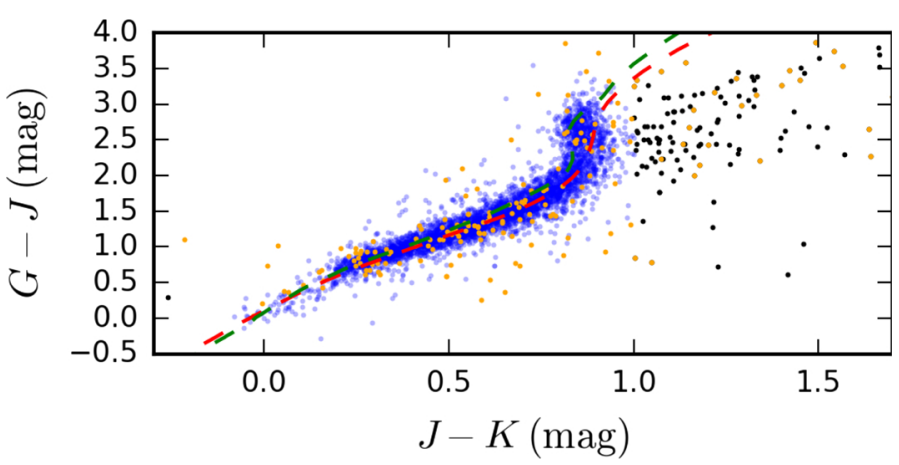

Fig. 15. Color-color diagram of the stellar XMMSL2 sources with Gaia and 2MASS counterparts. The sources represented as orange and black dots are flagged because of their known erroneous 2MASS photometry and their unusual colors, respectively. The red and green dashed curves represent various theoretical models of the main sequence; see text for details.

\subsection{Color-color diagram}

About $80 \%$ of the unique sources are covered by the 2MASS and Gaia catalogs, and we constructed color-color diagrams for those sources. Figure 15 shows that the sources are arranged in a well-defined streak. However, a few sources are located outside of the main distribution, many of which are known to have an erroneous 2MASS photometry. Many sources at the red end are classified as pre-main sequence stars by SIMBAD and their location in Fig. 15 is likely due to reddening. Since we do not perform any correction for extinction either in the optical or at X-ray wavelengths, we flag these sources in our catalog. Another possibility is an error in the optical magnitudes leading to incorrect colors, again, this motivates flagging these sources.

Figure 15 also shows two theoretical color-color relations for main sequence stars. We adopt color-color relations from Worthey \& Lee (2011) and Pecaut \& Mamajek (2013) to obtain the theoretical correlation between $G-J$ and $J-K$. The estimated theoretical color-color relation generally corresponds well with the observed distribution and differs only for the sources with 


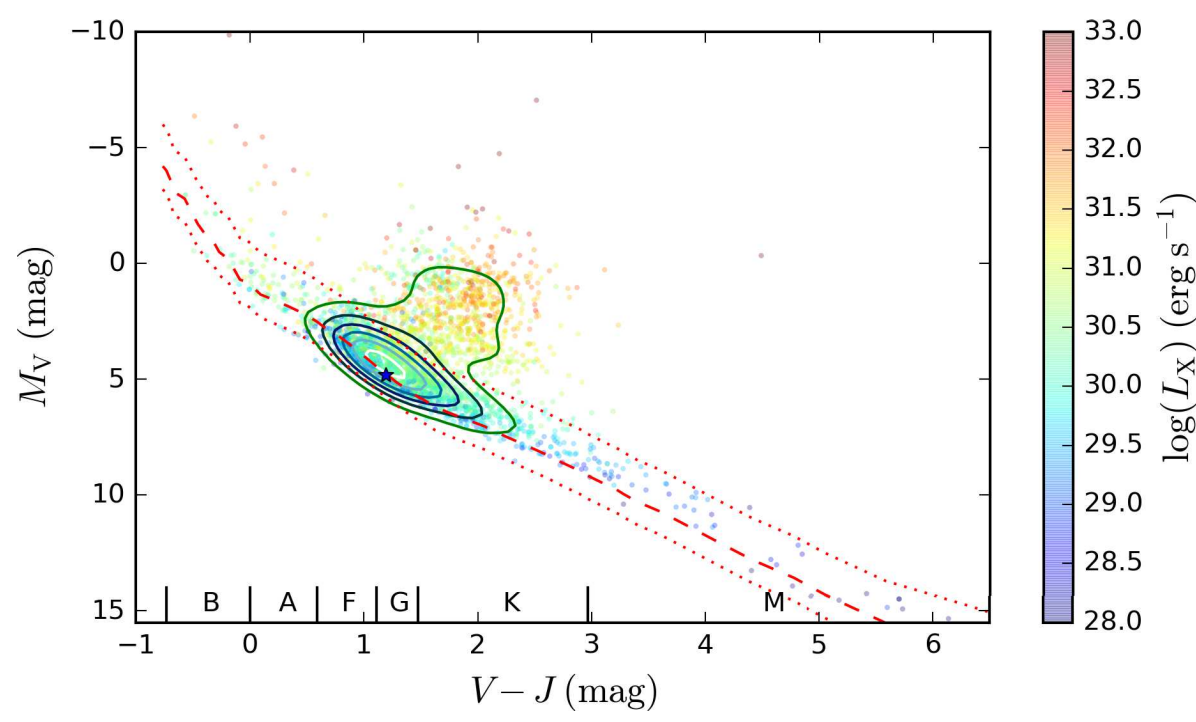

Fig. 16. Hertzsprung-Russell diagram of the stellar XMMSL2 sources with known parallax, the color scales with the logarithmic X-ray luminosity $\log \left(L_{\mathrm{X}}\right)$. The red dashed line represents the theoretical main sequence following Pecaut \& Mamajek (2013), and the dotted lines show the assumed width of the main sequence. The star marker represents the position of the Sun; we note that the Sun is less luminous than the stellar XMMSL2 sources. The density of the distribution is indicated by the colored lines. The ranges of the spectral types for dwarfs are given at the bottom of the figure.
$J-K \approx 0.8$. However, for these sources the predicted correlation between $G-J$ and $J-K$ substantially depends on the assumed model.

\subsection{Hertzsprung-Russell diagram}

About $57 \%$ of our sources with a unique stellar identification have trigonometric parallaxes, either from Gaia or the HIPPARcos catalog (ESA 1997), or in the Lepine and BrightStar catalogs. Figure 16 shows the Hertzsprung-Russell diagram (HRD) of these sources. We point out that this sample of stellar counterparts with trigonometric parallaxes is neither complete in brightness nor in volume (many optically faint sources currently lack parallaxes), however, Fig. 16 does contain all basic features known from an optically selected HRD. The main sequence covers a wide range $(-0.9 \mathrm{mag}<V-J<6 \mathrm{mag})$, hence the sample contains all spectral types from O-type stars down to dwarfs of spectral type M6V albeit sparsely populated at the limiting spectral types. Figure 16 contains only a small number of M-type dwarfs and some of them might actually be reddened K-type dwarfs, although late-type dwarfs show frequent and extreme flares. However, the fluxes at X-ray and optical wavelengths are low for these sources, and hence, the parallaxes are currently unknown. Furthermore, the complete sample also includes even later type stars, but these sources lack parallaxes and are mainly flagged because of the extreme color.

In the following, we assume sources to be main sequence stars if their absolute brightness $M_{\mathrm{V}}$ is in the range $M_{\mathrm{V} \text {,theo }}+1.0>M_{V}>M_{\mathrm{V} \text {,theo }}-1.8$, where $M_{\mathrm{V} \text {,theo }}$ is the theoretical absolute brightness of a main sequence star adopted from Pecaut \& Mamajek (2013). We find that $64 \%$ of the sources are dwarfs. However, not all of these sources need to be single stars, rather we expect many sources to be X-ray bright, active binaries. Also, the giant branch is clearly evident and we expect many of these sources to be RS CVn systems.

The HRD presented by Güdel (2004) contains about 2000 stars and shares many similarities with our HRD. However, the number of stars associated with the various categories differs strongly because our sample is drawn from a flux limited sample that is biased toward active systems, while Güdel (2004) congregated data from several studies. This also explains why we only find a few early-type stars and no separate population of premain sequence stars. Nevertheless, we expect that our complete sample contains some pre-main sequence stars, but these sources either lack parallaxes or are flagged because of reddening.

\section{5. $F_{X} / F_{\text {bol }}$-ratio}

Figure 17 shows the $F_{\mathrm{X}} / F_{\text {bol }}$ distribution of the stellar XMMSL2 sources as a function of the effective temperature (see Fig. 11 for the histogram of the distribution). We also include sources that are flagged in our catalog due to optical loading or their high $F_{\mathrm{X}} / F_{\text {bol }}$ values. The stars cover a wide range of activity, some sources have very high values of $F_{\mathrm{X}} / F_{\text {bol }}$, and we find that about $14 \%$ of the sources have an X-ray activity of $\log \left(F_{\mathrm{X}} / F_{\text {bol }}\right)>-2.5$, which is substantially higher than the saturation limit found in previous studies (e.g., Pizzolato et al. 2003). For many of these sources the RASS flux is up to 10 times fainter (see Fig. 12) and we expect them to be detected by XMM-Newton during a flare. We flag sources with persistent (XMMSL+ RASS) high X-ray flux since these are probably nonstellar sources. Furthermore, we flag strong transients and sources lying above the red dashed line in Fig. 17; the high X-ray activity of these sources is unlikely caused by flares. Almost $6 \%$ of the sources lie above the threshold of $\log \left(F_{\mathrm{X}} / F_{\text {bol }}\right)>-2.2$ that we use to calculate the magnitude cutoff. Since this magnitude cutoff is particularly relevant at both ends of the spectral type distribution, some true stellar identifications might be missed, especially in the M dwarf regime. At very low masses, sources are flagged owing to the extreme red colors and are missing in this figure.

The X-ray activity of the stars strongly depends on the spectral type and several known features are visible in Fig. 17. First, the onset of convection at about $7000 \mathrm{~K}$ is evident. Second, two distinct so-called fingers at $7000 \mathrm{~K}$ and $5000 \mathrm{~K}$ contain sources down to relatively low activity levels. Most sources in the first finger are F dwarfs, while the second finger mainly consists of late $\mathrm{G}$ and early $\mathrm{K}$ giants. Since the X-ray flux has to be above the detection limit, the sources with a very low activity level must have a high (apparent) bolometric flux. It is also evident from the color coding that the low activity sources are relatively bright in the optical and are either nearby, inactive dwarfs or distant, X-ray bright giants. Third, there are few early-type stars that have a low X-ray flux compared to their bolometric flux.

We show the sources with more than 17 counts (10\% of the samples) and, hence, with highly reliable X-ray fluxes as black 


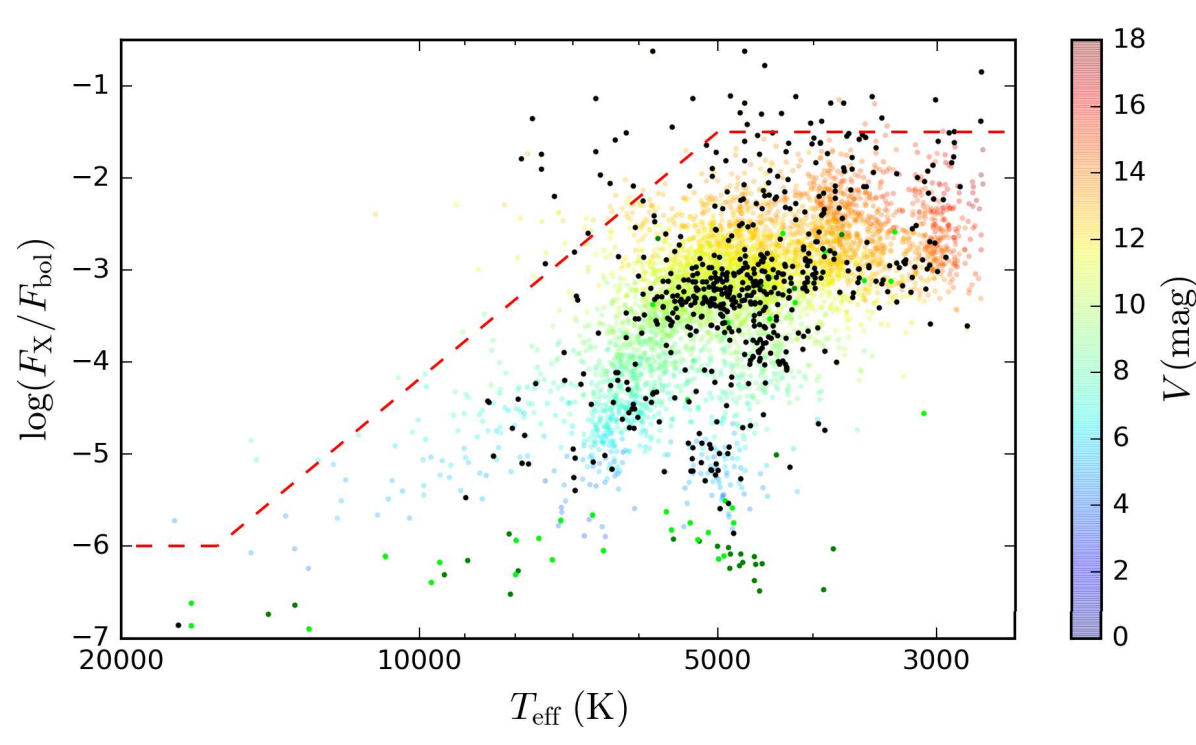

Fig. 17. $F_{\mathrm{X}} / F_{\text {bol }}$ distribution of the stellar XMMSL2 sources as a function of effective temperature. The colors scale with $V$ band magnitude. Sources that may be affected by optical loading are shown as green dots, while those sources with a RASS identification are indicated in light green, and those sources without RASS counterpart are shown as dark green dots. The black dots represent highly reliable XMMSL2 sources with more than 17 cts (about $10 \%$ of our sample). Sources lying above the red dashed line are flagged in our catalog as high X-ray activity during the slew. dots in Fig. 17. Most of these sources have $\log \left(F_{\mathrm{X}} / F_{\text {bol }}\right)<-3$, however, for some sources the contribution of the X-ray flux to the bolometric flux is extremely high. These sources could not be associated with a stellar counterpart brighter than the magnitude cutoff if the source had been detected at the XMMSL2 detection limit.

\section{Conclusions and outlook}

In this paper we present the first in-depth analysis of the stellar content of the XMM-Newton slew survey. In our approach the stellar XMMSL2 sources are identified by an automatic crossmatch of the XMMSL2 catalog with the Gaia DR1, 2MASS, and Tycho 2 catalogs; we expect that in the future all necessary stellar data may actually be adopted from the new Gaia releases. We tested and verified the reliability of our automatic identification procedure by a comparison with the individually classified EMSS sources and by a crossmatch of our stellar identifications with the CSC.

With our procedure a total of 6815 stellar counterparts were found for the 23252 XMMSL2 sources, after applying our cleaning procedures we identified 5920 XMMSL2 sources as high quality stellar X-ray sources corresponding to a stellar fraction of 25.5

further expect that 195 stellar classifications are spurious, i.e. they are erroneously attributed to a star. On the other hand, we expected 227 stellar identifications to be missed due to the chosen probability cutoff. Therefore our sample has a reliability of $96.7 \%$ and a completeness of $96.3 \%$. We further expected a small fraction of true stellar identifications to be missed due to our magnitude cutoffs. This is a significant improvement to the stellar classifications presented by Saxton et al. (2008), who had no Gaia data at their disposal and identified only 4231 of our 5920 stellar XMMSL2 sources as stars. Saxton et al. (2008) further gave a stellar identification for 863 XMMSL2 sources that we did not identify as stars with our procedure, however, we show that most of the sources are less reliable stellar counterparts due to their high $F_{\mathrm{X}} / F_{\text {bol }}$ values.

The XMMSL2 catalog contains stars of all spectral types and luminosity classes, however, most of the stellar XMMSL2 sources are - not unexpectedly - late-type dwarfs with an outer convection zone. Only about $75 \%$ of the XMMSL2 sources have a RASS identification, although the RASS catalog is deeper by a factor of about 2.5. Hence, a substantial portion of the stellar XMMSL2 sources are previously unknown X-ray sources caught in an active or flaring state. With procedures as developed in this paper we expect to be able to perform an online identification of the stellar sources in the upcoming eROSITA all-sky survey.

Acknowledgements. This research has made use of data obtained from XMMSL2, the Second XMM-Newton Slew Survey Catalogue, produced by members of the XMM SOC, the EPIC consortium, and using work carried out in the context of the EXTraS project ("Exploring the X-ray Transient and variable Sky", funded from the EU's Seventh Framework Programme under grant agreement no. 607452). Additionally, we used data from the European Space Agency (ESA) mission Gaia (https://www. cosmos.esa.int/gaia), processed by the Gaia Data Processing and Analysis Consortium (DPAC; https://www. cosmos.esa.int/web/gaia/dpac/consortium). Funding for the DPAC has been provided by national institutions, in particular the institutions participating in the Gaia Multilateral Agreement. Furthermore, this publication made use of data products from the Two Micron All Sky Survey, which is a joint project of the University of Massachusetts and the Infrared Processing and Analysis Center/California Institute of Technology, funded by the National Aeronautics and Space Administration and the National Science Foundation. Moreover, we used the VizieR catalog access tool and the SIMBAD database, operated at CDS, Strasbourg, France. The original description of the VizieR service was published in A\&AS 143, 23 and we have made use of data obtained from the Chandra Source Catalog, provided by the Chandra X-ray Center (CXC) as part of the Chandra Data Archive. SF acknowledge supports through the Integrationsamt Hamburg, the ZAV of Bundesagentur für Arbeit, and the Hamburg University, JR by DLR under grant 50 QR 1605 and PCS through the SFB 676 founded by DFG and by DLR under grant 50 OR 1706.

\section{References}

Agrawal, P. C., Rao, A. R., \& Sreekantan, B. V. 1986, MNRAS, 219, 225 Ayres, T. R. 2009, ApJ, 696, 1931

Berghoefer, T. W., Schmitt, J. H. M. M., Danner, R., \& Cassinelli, J. P. 1997, A\&A, 322, 167

Boller, T., Freyberg, M. J., Trümper, J., et al. 2016, A\&A, 588, A103

Carpenter, J. M. 2001, AJ, 121, 2851

Catura, R. C., Acton, L. W., \& Johnson, H. M. 1975, ApJ, 196, L47

Crawford, D. F., Jauncey, D. L., \& Murdoch, H. S. 1970, ApJ, 162, 405

ESA 1997, The Hipparcos and TYCHO catalogues. Astrometric and photometric star catalogues derived from the ESA Hipparcos Space Astrometry Mission (Noordwijk: ESA Publications Division) ESA SP, 1200

Dempsey, R. C., Linsky, J. L., Fleming, T. A., \& Schmitt, J. H. M. M. 1993, ApJS, 86, 599

Evans, I. N., Primini, F. A., Glotfelty, K. J., et al. 2010, ApJS, 189, 37

Favata, F., Micela, G., Orlando, S., et al. 2008, A\&A, 490, 1121

Fleming, T. A., Liebert, J., Gioia, I. M., \& Maccacaro, T. 1988, ApJ, 331, 958 
Gaia Collaboration (Brown, A. G. A., et al.) 2016a, A\&A, 595, A2

Gaia Collaboration (Prusti, T., et al.) 2016b, A\&A, 595, A1

Gioia, I. M., Maccacaro, T., Schild, R. E., et al. 1990, ApJS, 72, 567

Güdel, M. 2004, A\&ARv, 12, 71

Haisch, B., Schmitt, J. H. M. M., \& Rosso, C. 1991, ApJ, 383, L15

Hempelmann, A., Schmitt, J. H. M. M., Baliunas, S. L., \& Donahue, R. A. 2003, A\&A, 406, L39

Hoffleit, D., \& Jaschek, C. 1991, The Bright Star Catalogue

Høg, E., Fabricius, C., Makarov, V. V., et al. 2000, A\&A, 355, L27

Huensch, M., Schmitt, J. H. M. M., Schroeder, K.-P., \& Reimers, D. 1996, A\&A, 310,801

Huensch, M., Schmitt, J. H. M. M., \& Voges, W. 1998a, A\&AS, 132, 155

Huensch, M., Schmitt, J. H. M. M., \& Voges, W. 1998b, A\&AS, 127, 25

Jansen, F., Lumb, D., Altieri, B., et al. 2001, A\&A, 365, L1

Jordi, C., Gebran, M., Carrasco, J. M., et al. 2010, A\&A, 523, A48

Lépine, S., \& Gaidos, E. 2011, AJ, 142, 138

Linsky, J. L., \& Haisch, B. M. 1979, ApJ, 229, L27

Merloni, A., Predehl, P., Becker, W., et al. 2012, ArXiv e-prints [arXiv:1209.3114]

Pallavicini, R., Golub, L., Rosner, R., et al. 1981, ApJ, 248, 279

Pallavicini, R., Tagliaferri, G., \& Stella, L. 1990, A\&A, 228, 403

Pecaut, M. J., \& Mamajek, E. E. 2013, ApJS, 208, 9

Peres, G., Orlando, S., Reale, F., Rosner, R., \& Hudson, H. 2000, ApJ, 528, 537
Pevtsov, A. A., Fisher, G. H., Acton, L. W., et al. 2003, ApJ, 598, 1387 Pizzolato, N., Maggio, A., Micela, G., Sciortino, S., \& Ventura, P. 2003, A\&A 397, 147

Predehl, P. 2017, Astron. Nachr., 338, 159

Robrade, J., Schmitt, J. H. M. M., \& Favata, F. 2012, A\&A, 543, A84

Saxton, R. D., Read, A. M., Esquej, P., et al. 2008, A\&A, 480, 611

Schmitt, J. H. M. M. 1997, A\&A, 318, 215

Schmitt, J. H. M. M., \& Liefke, C. 2004, A\&A, 417, 651

Schmitt, J. H. M. M., Fleming, T. A., \& Giampapa, M. S. 1995, ApJ, 450, 392

Skrutskie, M. F., Cutri, R. M., Stiening, R., et al. 2006, AJ, 131, 1163

Stelzer, B., Schmitt, J. H. M. M., Micela, G., \& Liefke, C. 2006, A\&A, 460, L35

Stocke, J. T., Morris, S. L., Gioia, I. M., et al. 1991, ApJS, 76, 813

Vaiana, G. S., Cassinelli, J. P., Fabbiano, G., et al. 1981, ApJ, 245, 163

Vilhu, O. 1984, A\&A, 133, 117

Voges, W., Aschenbach, B., Boller, T., et al. 1999, A\&A, 349, 389

Walter, F., Charles, P., \& Bowyer, S. 1978, ApJ, 225, L119

Weisskopf, M. C., Tananbaum, H. D., Van Speybroeck, L. P., \& O’Dell, S. L. 2000, in X-Ray Optics, Instruments, and Missions III, eds. J. E. Truemper, \& B. Aschenbach, Proc. SPIE, 4012, 2

Weisskopf, M. C., Brinkman, B., Canizares, C., et al. 2002, PASP, 114, 1

Wenger, M., Ochsenbein, F., Egret, D., et al. 2000, A\&AS, 143, 9

Worthey, G., \& Lee, H.-c. 2011, ApJS, 193, 1 
Appendix A: Flowchart of the matching and cleaning procedure

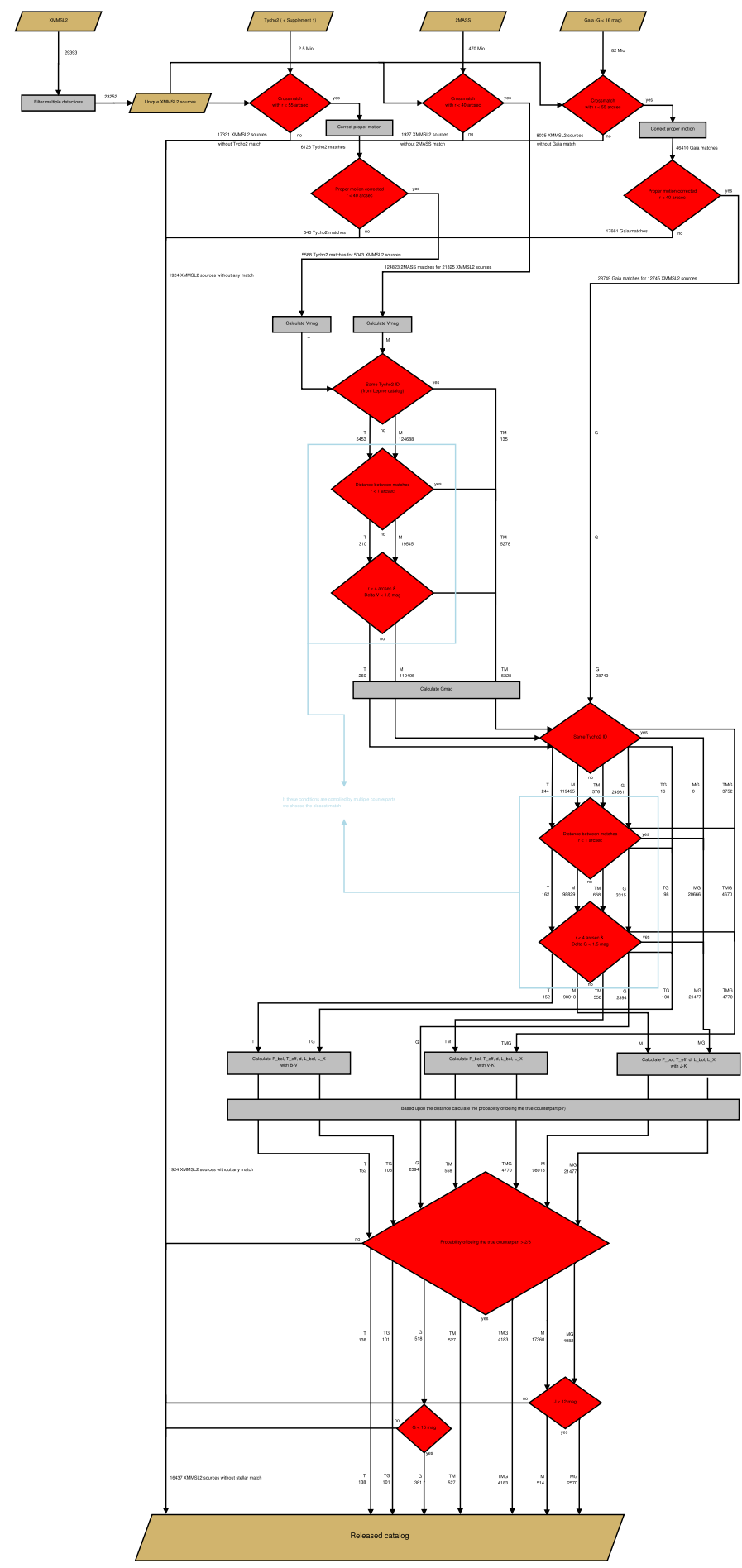

Fig. A.1. Flowchart of the matching procedure.

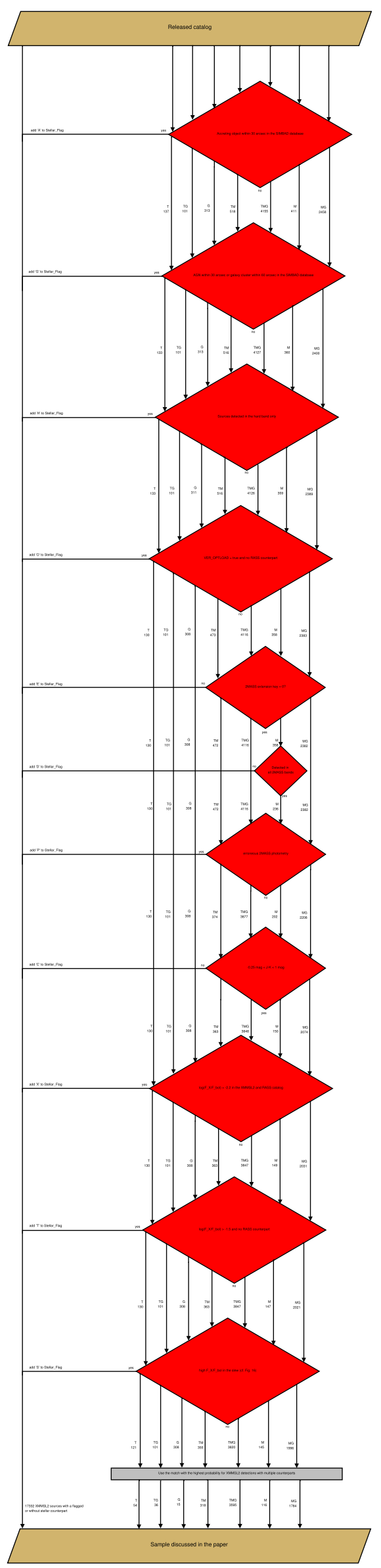

Fig. A.2. Flowchart of the cleaning procedure. 


\section{Appendix B: Column description}

We adopt all columns of the XMMSL2 catalog, described online $^{3}$, and extend further 57 columns defining our stellar counterpart. In the following we describe these columns. For measurements given in multiple catalogs, we define an order of priority from which catalog the values are adopted.

\section{XMMSLFX}

We convert the count rates given in XMMSL2 catalog into a 0.1 $2.4 \mathrm{keV}$ X-ray flux (cf. Sect. 3.1). We use the count rate of the total band if available, otherwise we adopt the count rate of the soft band or the hard band.

Units: $\mathrm{fW} / \mathrm{m}^{2}$

Order of priority: total band, soft band, hard band

\section{NumDet}

Number of detections of the XMMSL2 source

\section{NumMatch}

Number of stellar counterparts of the XMMSL2 source

\section{Priority}

For XMMSL2 sources with multiple counterparts, the counterparts are sorted by the matching probability "Prob_r". This column gives the ranking of the counterparts so that the identification with the highest matching probability is set to "Priority $=1$ ".

\section{Catalog}

Catalog of the stellar counterpart

G: Gaia catalog

T: Tycho 2 catalog

M: 2MASS catalog

L: Lepine catalog

B: BrightStar catalog

For counterparts given in multiple catalogs, multiple abbreviations are given, e.g., "TMG".

\section{r}

The distance $r$ between the position given in the XMMSL2 cata$\log$ and the proper motion corrected position of the stellar counterpart

Units: arcsec

Order of priority: Gaia catalog, Tycho2 catalog, Lepine catalog, BrightStar catalog, 2MASS catalog

\section{Prob $r$}

Matching probability as defined in Eq. (2) of the stellar counterpart

Units: \%

Order of priority: Lepine catalog, BrightStar catalog, Tycho2 catalog, 2MASS catalog, Gaia catalog

\footnotetext{
3 https://www . cosmos. esa.int/web/xmm-newton/xmmsl2-ug
}

\section{GaiaID}

Identifier of the Gaia catalog

\section{Tycho2ID}

Identifier of the Tycho 2 catalog

HR

Identifier of the BrightStar catalog

\section{PM}

Identifier of the SUPERBLINK catalog given in the Lepine catalog

2MASSID

Identifier of the 2MASS catalog

\section{MtchRA, MtchDE}

Proper motion corrected position of the stellar counterpart Units: degrees

Order of priority: Gaia catalog, Tycho 2 catalog, Lepine catalog, BrightStar catalog, 2MASS catalog

\section{e_MtchRA, e_MtchDE}

Statistical error on the position of the stellar counterparts if it is available

Units: mas

pmRA, pmDE, e_pmRA, e_pmDE

Proper motion and their statistical errors of the stellar counterpart

Units: mas $\mathrm{yr}^{-1}$

Order of priority: Tycho 2 catalog, Lepine catalog, BrightStar catalog

\section{BTmag, e_BTmag, VTmag, e_VTmag}

Magnitude $B_{T}$ and $V_{T}$ and their statistical errors adopted from the Tycho2 catalog.

Units: mag

\section{Bmag}

The $B$ magnitude is given in the Lepine catalog, but originates from the USNO catalog. It can also be estimated from the color $B-V$ given in the BrightStar catalog or from the Tycho 2 colors (ESA 1997).

Units: mag

Order of priority: Tycho 2 catalog, BrightStar catalog, Lepine catalog

\section{Vmag}

The $V$ magnitude is directly measured in the BrightStar catalog. In the Lepine catalog the $V$ magnitude is also given, but it is estimated from magnitudes of other photometric bands. We can estimate the $V$ magnitude from the Tycho 2 colors (ESA 1997) or 
extrapolate it from the color $J-K$ of the 2MASS catalog.

Units: mag

Order of priority: Tycho 2 catalog, BrightStar catalog, Lepine catalog, 2MASS catalog

\section{Gmag}

$\mathrm{G}$ magnitude adopted from the Gaia catalog.

Units: mag

Jmag, e_Jmag, Hmag, e_Hmag, Kmag, e_Kmag

Magnitudes in the $J, H$, and $K$ bands and the statistical errors adopted from the 2MASS catalog.

Units: mag

Qflg

Quality of the 2MASS magnitude measurement adopted from the 2MASS catalog (ph_qual).

\section{Rflg}

This flag describes which method has been applied to determine the magnitude for every 2MASS photometric band (rd_flg). If the flag contains a " 0 ", the source is not detected in that band. We flag these sources if they do not have an entry in another catalog.

\section{Bflg}

Number of sources for which the 2MASS magnitude is estimated at the same time (bl_flg).

\section{Cflg}

This flag indicates if the magnitude or the position of a 2MASS source is contaminated by a nearby source (cc_flg).

\section{Xflg}

This flag indicates if a 2 MASS point source lies within the boundaries of an extended 2MASS source (gal_contam).

\section{XSCID}

If a 2MASS point source is associated with an extended 2MASS source, this column gives the identifier of the extended source (ext_key).

plx, e_plx

Trigonometric parallax and their statistical errors if it is known Units: arcsec

Order of priority: Gaia catalog, HipParcos catalog, Lepine catalog, BrightStar catalog

\section{CCDM}

Identifier of the component of multiple star system adopted from the Tycho 2 or the BrightStar catalog

Order of priority: Tycho 2 catalog, BrightStar catalog

\section{SpType}

Spectral type adopted from the BrightStar or Lepine catalog Order of priority: BrightStar catalog, Lepine catalog

$\mathrm{mbol}$

We estimate the bolometric magnitude using Table 3 of Worthey \& Lee (2011). For the calculation we use different colors in the following order of priority.

Units: mag

Order of priority: $V-K, B-V, J-K$

\section{Fbol}

Given the bolometric magnitude, we estimate the bolometric flux.

Units: $\mathrm{fW} / \mathrm{m}^{2}$

\section{Teff}

Effective temperature given in Table 3 of Worthey \& Lee (2011) Units: $\mathrm{K}$

Order of priority: $V-K, B-V, J-K$

\section{VMag, Mbol}

Given the trigonometric parallax, we estimate the absolute magnitude.

Units: mag

Lbol, LX

Given the trigonometric parallax, we estimate the luminosity. Units: W

\section{StFlg}

Table B.1. Stellar flag.

\begin{tabular}{|c|c|c|c|}
\hline Sy. & Description & Definition & No. \\
\hline A & Accretor warning & $\begin{array}{l}\text { Accreting objects within } 30 \text { arcsec } \\
\text { in the SIMBAD database }\end{array}$ & 111 \\
\hline G & Extragalactic warning & $\begin{array}{l}\text { AGN within } 30 \text { arcsec or a galaxy } \\
\text { cluster within } 60 \operatorname{arcsec} \text { in the SIM- } \\
\text { BAD database }\end{array}$ & 116 \\
\hline $\mathrm{O}$ & Optical loading & $\begin{array}{l}\text { "VER_OPTLOAD" is true and no } \\
\text { RASS counterpart }\end{array}$ & 59 \\
\hline $\mathrm{E}$ & Extended source & $\begin{array}{l}\text { "ext_key" } \neq 0 \text { in the 2MASS cata- } \\
\log \end{array}$ & 21 \\
\hline $\mathrm{D}$ & Missing 2MASS detection & $\begin{array}{l}\text { 2MASS counterpart only and } \\
\text { "rd_flg" }=0 \text { in one band }\end{array}$ & 22 \\
\hline $\mathrm{P}$ & $\begin{array}{l}\text { Erroneous 2MASS pho- } \\
\text { tometry }\end{array}$ & $\begin{array}{l}\text { "ph_qual" }=X, U, F \text { or } E \text { in one } \\
\text { band }\end{array}$ & 467 \\
\hline $\mathrm{C}$ & Extreme color & $J-K<-0.25 \mathrm{mag}$ or $J-K>1 \mathrm{mag}$ & 311 \\
\hline $\mathrm{H}$ & Hard band detection only & $\begin{array}{l}\text { No values given in "RATE_B8" and } \\
\text { "RATE_B6" }\end{array}$ & 3 \\
\hline $\mathrm{X}$ & $\begin{array}{l}\text { Persistent high X-ray ac- } \\
\text { tivity }\end{array}$ & $\begin{array}{l}\log \left(F_{\mathrm{X}} / F_{\text {bol }}>-2.2 \text { in the }\right. \\
\text { XMMSL2 and RASS catalog }\end{array}$ & 61 \\
\hline $\mathrm{T}$ & Transient X-ray source & $\begin{array}{l}\log \left(F_{\mathrm{X}, \mathrm{XMMSL} 2} / F_{\text {bol }}>-1.5 \text { and no }\right. \\
\text { RASS counterpart }\end{array}$ & 22 \\
\hline$S$ & $\begin{array}{l}\text { High X-ray activity in the } \\
\text { slew }\end{array}$ & $\begin{array}{l}\text { Fractional contribution of the X-ray } \\
\text { flux to the bolometric flux in the } \\
\text { slew higher than usual for a source } \\
\text { with the specific effective tempera- } \\
\text { ture (cf. Fig. 17) }\end{array}$ & 123 \\
\hline
\end{tabular}


S. Freund et al.: The stellar content of the XMM-Newton slew survey

2RXSID

Identifier of the closest RASS counterpart up to a distance of 60 arcsec

$2 \mathrm{RXSr}$

Distance between the XMMSL2 source and the closest RASS counterpart

Units: arcsec

\section{RXSFX}

RASS flux of the closest RASS counterpart adopting the conversion factor defined by Schmitt et al. (1995)

Units: $\mathrm{fW} / \mathrm{m}^{2}$

\section{SBDID}

Identifier of the closest SIMBAD counterpart up to a distance of 30 arcsec

\section{SBDr}

Distance between the XMMSL2 source and the closest SIMBAD counterpart

Units: arcsec

\section{SBDotype}

Classification of the closest SIMBAD counterpart 